\title{
Structural Alignment Methods with Applications to Geospatial Ontologies
}

\author{
Isabel F. Cruz and William Sunna \\ Department of Computer Science \\ University of Illinois at Chicago
}

Short title: Structural Alignment Methods

Keywords: ontology alignment, geospatial ontologies, data integration, semantic web

Corresponding author: Isabel F. Cruz, Department of Computer Science, University of Illinois at Chicago, 851 S. Morgan St. (M/C 152), Chicago, IL 60607, USA. E-mail: ifc@cs.uic.edu. 


\begin{abstract}
We consider the problem of enabling interoperability and information sharing among geospatial applications that use ontologies to describe their concepts and the relationships among them. We present two fully automatic alignment methods that use the graph structures of a pair of ontologies to establish their alignment, that is, the semantic correspondences between their concepts. We have tested our methods on geospatial ontologies pertaining to wetlands and four other pairs that belong to a repository that has been used in the Ontology Alignment Evaluation Initiative (OAEI). Using these ontologies, we have compared the effectiveness (precision and recall) of our methods against the Similarity Flooding Algorithm that was proposed by others and show that for each of the tested ontologies one of our methods is at least as effective as their method. We have tuned the performance of our methods by introducing a greedy approach that reduces the number of concepts that get compared. This approach reduces runtime by approximately $30 \%$ with a minor compromise to the effectiveness of the results. To further validate our approach, we participated in the OAEI competition to align a pair of ontologies, each with a few thousand concepts.
\end{abstract}




\section{Introduction}

Heterogeneities in geospatial information systems are generally caused by modeling the same aspects of reality in different ways. This is mainly due to the fact that such systems are developed by domain experts who have limited or no communication among them. Our focus in this paper is on classification schemes that are commonly used in geospatial applications such as land use or wetlands. For example, in the case of land use, there are potentially dozens of different land use data classifications associated with counties and municipalities, thus hindering land use planning across the borders of the different jurisdictions (Wiegand et al., 2002). As for wetlands, organizations monitoring their data inventory have an interest in sharing data. However, the lack of standard classification has long been identified as an obstacle to the development, implementation, and monitoring of conservation strategies both at the national and regional levels (Dini et al., 1998). In other words, data integration, defined as the problem of combining data that resides in different sources so as to provide users with a unified view over these data (Lenzerini, 2002), becomes only feasible if correspondences can be established across the different classification schemes that are associated with one or more geographical areas.

The classification schemes used by geospatial applications are often just syntactic constructs that assume different formats (for example, tables), where the hierarchical characteristics that should be inherent to a classification scheme are not always explicitly represented (Wiegand et al., 2002). Such legacy formats are in sharp contrast with current standardization efforts, such as the Open Geospatial Consortium, ${ }^{1}$ which rely on ontologies to describe the domains of interest. Ontologies contain a conceptual description of data, consisting of the concepts used, their attributes, and the relationships between those concepts. In this paper, we model classification schemes as ontologies. A direct consequence is that hierarchies of concepts can be readily modeled using "is-a" relationships. In this context, establishing correspondences across classification schemes is tantamount to ontology matching or alignment in which semantic connections are established among the concepts of two ontologies: one being the source ontology and the other the target ontology. Ontologies also play an important role in data integration (Cruz and Xiao, 2005).

Ontology alignment encompasses a wide variety of techniques, which are concept-based, that is, geared to the matching of single concepts (Bergamaschi et al., 1999; Castano et al., 2001; Palopoli et al., 1998) or structure-based, that is, taking into account the structural organization of several

\footnotetext{
${ }^{1}$ http://www.opengeospatial.org/.
} 
concepts at a time (Melnik et al., 2002; Noy and Musen, 2001; Rodríguez and Egenhofer, 2003). Other approaches are instance-based, that is, they consider data associated with the ontological concepts (Doan et al., 2002; Fossati et al., 2006; Ichise et al., 2001). In this paper, we concentrate on the first two techniques.

As ontologies grow in size or in number, their alignment should ideally be automatic or require minimum user intervention. For this reason, the Ontology Alignment Evaluation Initiative (OAEI) (Euzenat et al., 2007) promotes the competition of automatic alignment methods in terms of their effectiveness (recall, precision, and F-measure) and efficiency (runtime). Every year, they build a repository of ontology sets. Each set contains a source ontology, a target ontology, and the reference alignment between them, as determined by domain experts. Several of these sets are released after the competition. This is an important "side effect" of the competition. In fact, while the number of web available ontologies increases every day, it is the availability of reference alignments that provides researchers with ways of testing and evaluating their alignment methods objectively. The effort needed to produce those reference alignments is however considerable, therefore examples from many domains of interest, such as the geospatial domain, are not currently available.

In this paper, we present two (fully) automatic structure-based methods: the Descendant's Similarity Inheritance (DSI) method, which uses the relationships between ancestor concepts, and the Sibling's Similarity Contribution (SSC) method, which uses the relationships between sibling concepts. In our previous work, we have developed the AgreementMaker system, which incorporates several alignment methods (Cruz et al., 2002, 2005, 2004) organized in layers. The DSI and SSC methods have been incorporated in our system.

We have chosen the example of wetlands for our geospatial application domain, because it motivates well the need for structure-based methods. We implemented our proposed methods and tested them against our previously developed concept-based technique (Cruz et al., 2007), which provides us with a base case. Users can select a similarity value (threshold) for our concept-based method, henceforth called base similarity. We have investigated the influence of the choice of the threshold value in terms of precision and recall. In addition, we tested our methods against the implementation of an existing structure-based algorithm, the Similarity Flooding (SF) algorithm (Melnik et al., 2002). Besides the wetlands ontologies, our experiments involve aligning four ontology sets from the OAEI. Our experiments show that at least one of our structure-based methods is as effective or better than both our base case method and the SF algorithm. 
To further validate our approach, we competed in the 2007 Ontology Alignment Evaluation Initiative (OAEI) competition with the DSI method (Sunna and Cruz, 2007b). In the absence of the availability of a geospatial track, we competed in a the biomedical track, a "general purpose track" (in the sense that the alignment methods cannot take advantage of prior knowledge of the domain) where the objective was the alignment of two large ontologies consisting of a couple of thousand concepts each. A total of seven alignment systems (including ours) competed in aligning the biomedical ontologies. The alignment of these ontologies included three test cases whose aim was respectively to get optimal values for F-measure, precision, and recall. Our system came respectively in third, fourth, and third places.

Having achieved satisfactory results in aligning the OAEI ontologies, our focus shifted to improving the performance of our automatic algorithms. To this end, we have tuned our methods by introducing a greedy approach that is selective to what concepts get compared. On average, our approach reduced runtime by around $30 \%$ with a small compromise in the quality of the alignment results.

This paper extends our previous work (Sunna and Cruz, 2007a) in several ways: we analyze the influence of the choice of the threshold value in terms of precision and recall; we present the results obtained in the OAEI 2007 competition; based on the results obtained, we propose a technique for improving the performance of our method; we study the influence of the improved performance technique on precision and recall.

The rest of this paper is organized as follows. In Section 2, we give an overview of related work in the area. We provide further details on the wetland ontologies and on their suitability for testing structure-based alignment methods in Section 3. We describe briefly our multi-layered approach to ontology alignment as implemented by our alignment system in Section 4. In Section 5, we describe the $S F$ algorithm and present our automatic structure-based methods, DSI and SSC, that support the first layer of our system. We describe the results of applying our methods to five ontology sets including the wetlands set in Section 6. In Section 7, we discuss the influence of the base similarity threshold in precision and recall. We present the results of our participation in the 2007 OAEI competition in Section 8. In Section 9, we present our methodology for enhancing the performance of our automatic methods and the results of applying this enhancement to the alignment of the OAEI ontology sets. Finally, in Section 10, we draw conclusions and outline future work. 


\section{Related Work}

In their survey paper, Shvaiko and Euzenat (Shvaiko and Euzenat, 2005) provide a comparative review of recent schema and ontology matching techniques, which they classify as concept- or structure-based (respectively, element-level or structure-level in their terminology). In the conceptbased category, the techniques are, for example, string-based (e.g., edit-distance), language-based (e.g., tokenization), constraint-based (e.g., types, attribute cardinality) or use linguistic resources. An example of the last include the use of domain specific thesauri or of common knowledge thesauri, for example, a lexical database such as WordNet. ${ }^{2}$ The use of a resource that is not included in the ontologies to align, such as the use of thesauri, make a technique external (as opposed to internal). In the structured-based category, the techniques are, for example, graph-based (e.g., based on node or graph comparisons), taxonomy-based, which are based on particular kinds of relationships between the nodes (e.g., "is-a" relationships), or model-based, which are based on model theoretic semantics (e.g., description logic reasoning techniques). Their survey considers that concept-based techniques are not semantic, because "semantics is usually given in a structure," however, we consider the use of semantically organized thesauri such as WordNet to be semantic. Likewise, we consider the taxonomy-based techniques as semantic, given the semantics associated with relationships among components in a taxonomy.

The alignment techniques that we describe in this paper are either concept- or structure-based. In particular, our word comparison is both language-based and linguistic, the latter being both external and semantic. Our structure-based techniques are graph-based and because of our the taxonomic nature of our ontologies are also taxonomy-based. In the rest of this section, we outline several ontology matching approaches and systems and describe also related work on measuring similarity of ontological structures.

OLA (Owl-Lite Alignment) is an alignment tool for ontologies expressed in OWL (Euzenat et al., 2005). OLA first builds graphs from the ontologies and then applies a similarity model between concepts of the ontologies being aligned (Euzenat and Valtchev, 2004), which uses concept-based (string comparison and lexical comparison using WordNet) and structure-based techniques and supports both manual mappings and automated mappings. OLA tries to achieve the highest level of automation, by letting users provide a minimal set of parameters at the initial steps of the alignment process and then leaving it to the tool to end the alignment. Similarly to our approach,

\footnotetext{
${ }^{2}$ http://www.wordnet.com.
} 
they consider contributions of the neighbors (but only in the case of neighbors of the same type). Given the emphasis of OLA on OWL, it is difficult to compare our approach with theirs. For better results their approach needs to be semi-automatic, whereas our approach is designed to be fully automatic and can take advantage of the graph structure being a tree.

RiMOM (Risk Minimization based Ontology Mapping) uses conditional probabilities to model the mappings between two concepts and based on them defines candidate mappings. Finding the optimal mapping is formalized as finding the "action with minimal risk" (Tang et al., 2006). RiMOM considers both metadata and instances. For each possible mapping category (e.g., stringbased, taxonomy-based) they discover the optimal mappings independently of the other categories. Finally, they combine the results obtained for the different categories, by creating a combined value for each candidate mapping. The system can be run in an automatic or semi-automatic mode and uses an iterative process to arrive at a final mapping table. The mapping categories include string-based (edit-distance), lexical (including NLP techniques), constraint-based, linguistic (using a lexical and a statistical similarity dictionaries). As for structure-based techniques, they use a taxonomic approach. However, they appear to only consider the immediate super- and sub-concepts of a node.

Another approach for ontology alignment proposes the use of different mapping agents that establish semantic bridges between the concepts in the source and target ontologies (Silva et al., 2005). When conflicts arise, they are solved by a negotiation process among the agents. This negotiation takes into account the confidence values evaluated for each semantic bridge and the effort associated with the change on those values due to the negotiation process. In our AgreementMaker system (Cruz et al., 2007), which is formed of several layers that support different alignment methods, it is up to the final layer, the consolidation mapping layer to apply a priority scheme that is set by the domain expert, so as to solve conflicts among mappings produced by the different layers.

The Similarity Flooding algorithm is a structural technique, which can be used in matching a variety of data structures, called models (Melnik et al., 2002). Models can be data schemas, data instances, or a mixture of both. In their approach, models are converted to directed labeled graphs. For their algorithm to work, they rely on the fact that concepts from the two graphs are similar when their adjacent concepts on the graphs are similar. The algorithm starts by obtaining initial mappings between concepts in the two input graphs using a string matching function that returns initial similarities between matched concepts. Having established the initial mappings, the algorithm proceeds iteratively to establish more mappings between other concepts based on 
the assumption that whenever any two concepts in the input models match with some similarity measure, the similarity of their adjacent concepts increases. The iterations continue "flooding" the similarities across the concepts in the graphs until a fixed point is reached where similarity measures for all concepts have been stabilized. Of the matching techniques that we surveyed, this one is the closest to our vision of what a structure level approach should be, hence we have implemented their algorithm so as to compare its results with those of the methods that we propose in this paper.

The subject of measuring similarity of ontological structures (and of proposing metrics for this purpose) is related to the problem of ontology alignment. However, methods for measuring the similarity of ontologies return a value and methods for ontology alignment return a set of alignments between concepts. Having stated this difference, both methods need to establish comparisons between concepts. Next, we describe briefly three methods for measuring similarity of ontological structures, which hold similarities with the methods we use.

The first similarity method compares hierarchical ontologies and considers concepts and relations, using the notion of semantic cotopy, which, given a concept, returns all its super- and sub-concepts, thus taking into account the structure of the ontologies (Maedche and Staab, 2002). However, there is no amortized effect depending on the distance of those concepts. The second similarity method proposes an even more comprehensive similarity method that combines two components: lexical and taxonomic (which also uses the semantic cotopy notion) (Dellschaft and Staab, 2006). The third similarity method converges even for cyclic ontologies (as is the case with the $S F$ algorithm) such as those that may occur when OWL-Lite is used (Euzenat and Valtchev, 2004).

\section{Geospatial Application}

In this section, we describe a geospatial application for wetlands, which provides the motivation for performing structure-based alignment between ontologies. In defining wetlands, the United States adopts the Cowardin Wetland Classification System (Cowardin et al., 1979). In contrast, European nations use the International Ramsar Convention Definition (www.ramsar.org) and South Africa uses the National Wetland Classification Inventory (Dini et al., 1998). Because of the need for regionalization, it is difficult to have a standardized classification system between nations and also between regions of a country with a large geographic area (Cowardin et al., 1979).

[ Figure 1 to be placed about here. ] 
Figure 1 shows the Cowardin Wetland Classification System. As can be seen from the figure, there are several leaves in this hierarchy that are similar, even if their ancestor nodes are different. A similar observation can be made about the classification schemes for other wetlands systems. Therefore, one of the main challenges in aligning automatically any two wetland ontologies is the possibility of producing misleading mappings between concepts with the same name, which are however classified under non-corresponding categories. That is, establishing base similarities between concepts of the source ontology and concepts of the target ontology will not be sufficient to achieve a high degree of precision in relating concepts in the two ontologies. We illustrate further this point with Figure 2, where the source ontology (on the left) describes part of the Cowardin classification system and the target ontology (on the right) describes part of the South African classification system. When calculating the base similarities between concepts of the two ontologies, the concept Reef that belongs to the Intertidal wetlands subsystem in the source ontology, will yield a base similarity measure of $100 \%$ relative to the concept Reef that belongs to the Intertidal wetland subsystem in the target ontology. Furthermore, it will also yield a base similarity measure of $100 \%$ with the concept Reef that belongs to the Subtidal wetlands subsystem in the target ontology. However, if we consider the ancestors of the nodes, hence the structure of the graph, we will be able to find the correct mappings.

\section{[ Figure 2 to be placed about here. ]}

\section{AgreementMaker Framework}

We have been working on a framework that supports the alignment of two ontologies (Cruz et al., 2002, 2005, 2004, 2007). In our framework, we incorporate several alignment techniques. Each technique is embedded in a mapping layer (Cruz et al., 2007). Our mapping layers use concept-based alignment techniques (first layer) and structure-based alignment techniques (first and third layers). In addition, domain experts can use their knowledge and contribute to the alignment process in the manual layer (second layer) and in the deductive layer (third layer). In the third layer, alignments are automatically propagated along the ontologies using deductive rules, but manual input is needed when the process stops (Cruz et al., 2004). The fourth layer applies a priority scheme that is set by the domain expert, so as to solve conflicts among alignments produced by the different layers. The base similarity method and the structure-based methods were incorporated in the first layer. The motivation behind our multi-layer framework is to allow for the addition of as many mapping 
layers as possible in order to capture a wide range of relationships between concepts.

We have developed a system, the AgreementMaker, which implements our approach. It maps ontologies expressed in XML, RDFS, OWL, or N3. The user interface of the AgreementMaker displays the two ontologies side by side as shown in Figure 3, which are in this case two land use ontologies.

\section{[ Figure 3 to be placed about here. ]}

After loading the ontologies, the domain expert can start the alignment process by mapping corresponding concepts manually or invoking procedures that map them automatically (or semiautomatically). The mapping information is displayed in the form of annotated lines connecting the matched nodes. There are two types of annotations: textual, which describe the type of mapping (e.g., exact, subset, superset), and numerical, which capture the similarity between the related concepts (e.g., 75\%). The color of the lines represents the mapping layers that generate them (e.g., blue for the first layer, green for the second layer). The user decides what colors are assigned to which layers. To increase the clarity of the display, the user can specify a similarity threshold so that only the lines that have similarity values greater or equal to a selected threshold are displayed. In addition, a maximum number of lines (as associated with each concept) can be specified. The display of all the mappings that are obtained by the different layers can also lead to cluttering of the display. To further improve readability, we allow users to hide the results of any of the mapping layers and to redisplay them as desired.

Many choices were considered in the process of displaying the ontologies and their relationships (Cruz et al., 2007). We have extensively tested our user interface with GIS specialists who are knowledgeable of land use problems.

\section{$5 \quad$ Automatic Similarity Methods}

In order to achieve a high level of confidence in performing the automatic alignment of two ontologies, a thorough understanding of the concepts in the ontologies is highly desired. To this end, we propose methods that investigate the ontology concepts prior to making a decision on how they should be mapped. We consider not only the labels and definitions of the ontology concepts, but also the relative positions of the concepts in the ontology tree. In the first mapping layer, the user can select one of the following three matching methods: (1) base similarity only; (2) base similarity

followed by the Descendant's Similarity Inheritance (DSI) method; (3) base similarity followed by 
the Sibling's Similarity Contribution (SSC) method. Both the DSI and the $S S C$ methods, which are structure-based, have been introduced to enhance the alignment results that were obtained from using the base similarity method, which is concept-based (Cruz et al., 2007). Any other base similarity could have been used here (Resnik, 1995; Lin, 1998), but we would like to point out that our emphasis is on structure-based algorithms and on how they can improve upon a conceptbased method. Therefore, we have used our own base similarity method as the first step to all the structure-based algorithms we consider, including the Similarity Flooding algorithm of Melnik et al. (Melnik et al., 2002).

\subsection{Base similarity method}

The very first step in our approach is to establish initial mappings between the concepts of the source ontology and the concepts of the target ontology. This is achieved by using a similarity function and applying it to two concepts (one in each ontology). If the returned value is greater or equal to a threshold set up by the domain expert, then the two concepts match. The base similarity value is semantic in that it is determined with the help of a dictionary.

In what follows, we present the details of finding the base similarity between a concept in the source ontology and a concept in the local ontology:

- Let $S$ be the source ontology and $T$ be the target ontology.

- Let $C$ be a concept in $S$ and $C^{\prime}$ be a concept in $T$.

- Use function base_sim $\left(C, C^{\prime}\right)$ that yields a similarity measure $M$, such that $0 \leq M \leq 1$.

- Given threshold value $T H, C^{\prime}$ is matched to $C$ when base_sim $\left(C, C^{\prime}\right) \geq T H$.

- For every concept $C$ in $S$, define the mapping set of $C$, denoted $M S(C)$, as the set of concepts $C^{\prime}$ in $T$ that are matched to $C$ (i.e., base_sim $\left.\left(C, C^{\prime}\right) \geq T H\right)$.

The following steps determine the similarity between two concepts $C$ and $C^{\prime}$ by looking at their labels, label $(C)$ and $\operatorname{label}\left(C^{\prime}\right)$ :

- If $\operatorname{label}(C)$ is identical to the $\operatorname{label}\left(C^{\prime}\right)$, then return a similarity of 1 .

- Else, apply the treat_string function on $\operatorname{label}(C)$ and $\operatorname{label}\left(C^{\prime}\right)$; this function separates a composite string that contains multiple words into its components. For example, in an ontology 
that contains concepts related to types of weapons, if label $(C)=$ "air-to-air-missile", then the string of the label will be converted to "air to air missile" after applying the function. Similarly, if label $(C)=$ "ServerSoftware" in an ontology that contains concepts related to computers and networks, it will be converted to "Server Software" after applying the treat_string $(\operatorname{label}(C))$ function. The function also takes care of words separated by underscore "." characters.

- After applying the treat_string function to label $(C)$ and $\operatorname{label}\left(C^{\prime}\right)$, check if the two resulting labels are identical; if true, return a similarity measure of 1 , else proceed to the next step.

- Remove all "Stop words" (such as, "the", "a", "to") from label $(C)$ and label $\left(C^{\prime}\right)$.

- Retrieve the definitions of every remaining word in the labels from the WordNet dictionary. For label $(C)$, concatenate the definition of all the words that make up the label into the string $D$. For label $\left(C^{\prime}\right)$, concatenate the definitions of all the words that make up the label into the string $D^{\prime}$.

- Apply the stemming algorithm (Hull, 1996) on every word in $D$ and $D^{\prime}$. On a high level, the algorithm traces words back to their roots, for example it reduces the words "directed" and "directing" to "direct" and "direct" respectively. In this way, the two words become comparable to each other.

- Let len $(D)$ be the number of words in the string $D$, len $\left(D^{\prime}\right)$ be the number of words in the string $D^{\prime}$, and common_count $\left(D, D^{\prime}\right)$ be the number of unique common words between $D$ and $D^{\prime}$. Then, compute base_sim $\left(C, C^{\prime}\right)$ as: $\frac{2 \times \operatorname{common}_{-} \operatorname{count}\left(D, D^{\prime}\right)}{\operatorname{len}(D)+\operatorname{len}\left(D^{\prime}\right)}$. For example, the words "apple" and "orange" share just two words in their definitions, namely "fruit" and "yellow" and they have respectively 10 and 5 words in their definitions, therefore their base similarity is 0.27 . The definitions of the words "car" and "automobile" are identical in the dictionary, therefore the base similarity value is 1 .

- If base_sim $\left(C, C^{\prime}\right) \geq T H$, then add $C^{\prime}$ to $M S(C)$.

Our geospatial example of Section 3, which was illustrated in Figure 2 points to the need to consider not only nodes in isolation but to take into account the structure of the taxonomies involved. The following two methods reconfigure the base similarity between concepts based respectively on the concepts of which these nodes are descendants or siblings in the taxonomy. 


\subsection{Descendant's Similarity Inheritance (DSI) method}

We define the $D S I$ reconfigured similarity between a concept $C$ in $S$ and a concept $C^{\prime}$ in $T$ as $D S I \_s i m\left(C, C^{\prime}\right)$. In what follows, we present the details on how to determine $D S I_{-} \operatorname{sim}\left(C, C^{\prime}\right)$ :

- Let path_len_root $(C)$ be the number of edges between the concept $C$ in $S$ and the root of the ontology tree $S$. For example, in Figure 4, path_len_root $(C)=2$. Similarly, we define path_len_root $\left(C^{\prime}\right)$ with respect to $T$. For example, in Figure 4, path_len_root $\left(C^{\prime}\right)=2$.

- Let parent $(C)$ be the ith concept from the concept $C$ to the root of the source ontology $S$, where $0 \leq i \leq$ path_len_root $(C)$. Similarly define parent $_{i}\left(C^{\prime}\right)$ with respect to $T$. For example, in Figure 4, parent $_{1}(C)=B$ and parent $_{1}\left(C^{\prime}\right)=B^{\prime}$.

- Define $M C P$ as the main contribution percentage, which is the fraction of the similarity measure between $C$ and $C^{\prime}$ that will be used in determining the overall $D S I \_\operatorname{sim}\left(C, C^{\prime}\right)$.

- Compute $D S I_{-} \operatorname{sim}\left(C, C^{\prime}\right)$ as follows:

$$
\left.M C P \cdot b a s e \_s i m\left(C, C^{\prime}\right)+\frac{2(1-M C P)}{n(n+1)} \sum_{i=1}^{n}(n+1-i) \text { base_sim }\left(\operatorname{parent}_{i}(C), \text { parent }_{i}\left(C^{\prime}\right)\right)\right)
$$

where $n=\min \left(\right.$ path_len_root $(C)$, path_len_root $\left.\left(C^{\prime}\right)\right)$

- If $D S I_{-} \operatorname{sim}\left(C, C^{\prime}\right) \geq T H$, then add $C^{\prime}$ to $M S(C)$.

[ Figure 4 to be placed about here. ]

The main characteristic of the DSI method is that it allows for the parent and in general for any ancestor of a concept to play a role in the identification of the concept. Intuitively, the parent of a concept should contribute more to the identity of the concept than its grandparent. This is achieved by setting a relatively high value to $M C P$. The grandparent concept contributes more than the great grandparent, and so on, until the root is reached. This can be demonstrated by considering the example in Figure 4. In the figure, we show how the DSI similarity is determined between the concept $C$ in the source ontology $S$ (shown left) and the concept $C^{\prime}$ in the target ontology $T$ (shown right) when applying the $D S I$ method using an $M C P$ value of $75 \%$. The $D S I$ similarity is determined by adding $75 \%$ of the base similarity between $C$ and $C^{\prime}$ to $17 \%$ of the base similarity of their immediate parents $\left(B\right.$ and $\left.B^{\prime}\right)$ and finally to $8 \%$ of the base similarity of their grandparents $\left(A\right.$ and $A^{\prime}$ ). Experiments have shown that $75 \%$ for the value of the $M C P$ factor 
works well (in fact, any values in that neighborhood performed similarly). The following example illustrates just one such case.

Considering the case of Figure 2, the base similarity between the concepts Intertidal in the source ontology and the concept Subtidal in the target ontology is $37 \%$. The base similarity between the concepts Marine in the source ontology and the concept Marine in the target ontology is $100 \%$. When applying the $D S I$ method with an $M C P$ value of $75 \%$, the $D S I$ similarity between the concept Reef that belongs to the Intertidal wetland subsystem in the source ontology and the concept Reef that belongs to the Subtidal wetland subsystem in the target ontology will be $88 \%$. Applying the DSI method again between the concept Reef that belongs to the Intertidal wetland subsystem in the source ontology and the concept Reef that belongs to the Intertidal wetland subsystem in the target ontology will yield a similarity of $100 \%$. Therefore, we conclude that the last match is the best one (in fact the optimal one). This is just one example that shows how the DSI method can be useful in determining more accurate similarity measures between concepts.

\subsection{Sibling's Similarity Contribution (SSC) method}

In this method, siblings of a concept contribute to the identification of the concept. This may further enhance the quality of the automatic alignment process. Similarly to the DSI method, the $S S C$ method reconfigures the base similarities between concepts. We define the $S S C$ similarity between a concept $C$ in $S$ and a concept $C^{\prime}$ in $T$ as $S S C \_\operatorname{sim}\left(C, C^{\prime}\right)$. In what follows, we present the details on how to determine this similarity.

- Let sibling_count $(C)$ be the number of sibling concepts of concept $C$ in $S$. For example, in Figure 5, sibling_count $(C)=2$.

- Let sibling_count $\left(C^{\prime}\right)$ be the number of sibling concepts of concept $C^{\prime}$ in $T$. For example, in Figure 5, sibling_count $\left(C^{\prime}\right)=3$.

- Let $S S(C)$ be the set of all the concepts that are siblings of $C$ in $S$ and $S S\left(C^{\prime}\right)$ be the set of all the concepts that are siblings of $C^{\prime}$ in $T$.

- Let $S_{i}$ be the $i$ th sibling of concept $C$ where $S_{i} \in S S(C)$, and $1 \leq i \leq \operatorname{sibling}$ _count $(C)$.

- Let $S_{j}^{\prime}$ be the $j t h$ sibling of concept $C^{\prime}$ where $S_{j} \in S S\left(C^{\prime}\right)$, and $1 \leq j \leq$ sibling_count $\left(C^{\prime}\right)$.

- Define $M C P$ as the main contribution percentage, which is the fraction of the similarity measure between $C$ and $C^{\prime}$ that will be used in determining the overall $S S C \_\operatorname{sim}\left(C, C^{\prime}\right)$. 
- If both $S S(C)$ and $S S\left(C^{\prime}\right)$ are not empty, define $S S C_{-} \operatorname{sim}\left(C, C^{\prime}\right)$ as follows:

$$
M C P \cdot \text { base_sim }\left(C, C^{\prime}\right)+\frac{1-M C P}{n} \sum_{i=1}^{n} \max \left(\text { base_sim }\left(S_{i}, S_{1}^{\prime}\right), \ldots, \operatorname{base\_ sim}\left(S_{i}, S_{m}^{\prime}\right)\right)
$$

where $n=$ sibling_count $(C)$ and $m=$ sibling_count $\left(C^{\prime}\right)$.

- If $S S C_{-} \operatorname{sim}\left(C, C^{\prime}\right) \geq T H$, then add $C^{\prime}$ to $M S(C)$.

[ Figure 5 to be placed about here. ]

The main characteristic of the $S S C$ method is that it allows for the siblings of a given concept to play a role in the identification process of the concept. In Figure 5 we show how the $S S C$ similarity is determined between the concept $C$ in the source ontology $S$ (shown on the left) and the concept $C^{\prime}$ in the target ontology $T$ (shown on the right) when applying the $S S C$ method with an $M C P$ value of $75 \%$. The $S S C$ similarity is determined by adding $75 \%$ of the base similarity between $C$ and $C^{\prime}$ to (1) $12.5 \%$ of the maximum base similarity between $D$ and $D^{\prime}, D$ and $E^{\prime}$, and $D$ and $F^{\prime}$ and to (2) $12.5 \%$ of the maximum base similarity between $E$ and $D^{\prime}, E$ and $E^{\prime}$, and $E$ and $F^{\prime}$. Like for the DSI method, the value of $75 \%$ for the $M C P$ factor was found to work well in practice.

\subsection{Similarity Flooding algorithm}

The Similarity Flooding $(S F)$ exploits the structure of the schemas to establish correspondences between their elements (Melnik et al., 2002). We describe briefly how it works. First, the two input schemas are converted into directed labeled graphs as shown in the example of Figure 6.

\section{[ Figure 6 to be placed about here. ]}

Initial correspondences between the elements of the two graphs are established using string matching techniques. Let us assume that given the similarity values found, correspondences between pairs of values were established as follows: $(S 1, T 1),(S 2, T 2),(S 3, T 2)$, and $(S 2, T 3)$.

Following this initial step, a pairwise connectivity graph is derived as shown in Figure 7.

\section{[Figure 7 to be placed about here. ]}

The graph contains nodes that represents the matching pairs, which are connected according to the structural relationships in the original input graphs. For example, the node representing the correspondence between $S 1$ and $T 1$ is connected to the node representing the correspondence 
between $S 2$ and $T 2$ because $S 1$ is connected to $S 2$ in the source input graph, and $T 1$ is connected to $T 2$ in the target input graph. The $S F$ algorithm proceeds by propagating similarity values between neighbors in multiple iterations. Each node in the graph divides its propagated similarity values equally among its neighbors. For example, in Figure $7,(S 1, T 1)$ propagates half of its similarity to $(S 2, T 2)$ and the other half to $(S 3, T 2)$. Since node $(S 2, T 2)$ has only one neighbor $(S 1, T 1)$, all of its similarity value is propagated to it. After this process, the similarities that accumulate at each node get normalized by dividing them by the maximum similarity value in the graph. This process is repeated until a least fixed point is reached.

\section{Experimental results}

To validate our approach from the point of view of effectiveness and efficiency, we have aligned the two wetlands ontologies mentioned in Section 3 using our own methods: base similarity, DSI, and $S S C$. We have also used our implementation of the Similarity Flooding algorithm (Melnik et al., 2002). In addition, to further evaluate our methods, we run experiments on the alignment of four sets of ontologies provided by the Ontology Alignment Evaluation Initiative (OAEI) (Sure et al., 2004). Of these, the first set contains two ontologies describing classifications of various weapon types, the second set contains two ontologies describing attributes of people and pets, the third set contains two ontologies describing classifications of computer networks and equipments, and the fourth set contains general information about Russia. Each set contains a source ontology, a target ontology, and the expected alignment results between them. Table 1 displays the depth and number of concepts in the five ontology sets we consider.

\section{[ Table 1 to be placed about here. ]}

We conducted experiments to determine the efficiency (runtime) of the four methods (base similarity, DSI, SSC, and Similarity Flooding) for the previously mentioned five ontology sets. We have implemented all the methods using Java on a 1.6 GHz Intel Centrino Duo with 1GB of RAM, running Windows XP. The results are shown in Table 2.

The runtime for the DSI, SSC, and Similarity Flooding algorithms include the runtime for the base similarity method, because that method is run first in all cases. Therefore, the base similarity algorithm takes the least amount of time. Examining the remaining results, the DSI method has the best runtime performance for four of the test cases, while the Similarity Flooding algorithm has 
the best runtime performance for one test case only. The $S S C$ method has the worst performance in three test cases while it performs better than the Similarity Flooding algorithm in two test cases. The $S S C$ method depends on the number of siblings for a given concept, therefore the larger the number of siblings the worse it performs. In other words, if the ontology trees are wide, then the performance of $S S C$ will suffer. Similarly, the runtime of the DSI method degrades for deep ontology trees. In Section 9, we present a greedy algorithm that enhances the performance of our methods.

To compare the effectiveness of the four methods, we started by aligning the set of ontologies for the wetlands as described in Section 5.2 and did the same for the other four sets of ontologies. In the wetlands example, we have captured the number of discovered relations between the concepts of the source ontology (Cowardin) and the concepts of the target ontology (South African) for each method. Each relationship represents a mapping from a concept $C$ in the source ontology $S$ to a target ontology concept $C^{\prime} \in M S(C)$ with the highest similarity measure. We note that there may be concepts in $S$ that are not mapped to any concepts in the target ontology (corresponding to an empty mapping set). Also, there may be more than one concept in $S$ that maps to the same concept $C^{\prime}$. After capturing the discovered relations, we count how many of these relations are valid when compared with the expected alignment results. Having determined the number of valid relations, we calculate both the precision and recall values. Precision is calculated by dividing the number of discovered valid relations by the total number of discovered relations and recall is calculated by dividing the number of discovered valid relations by the total number of valid relations as provided by the expected alignment results.

In the alignment of the wetland ontologies, the DSI method yielded slightly higher precision and recall values than the Similarity Flooding algorithm which in turn yielded higher values than the SSC method. Overall, these three methods significantly enhanced the precision and recall values obtained by applying the base similarity method only. Table 3 shows the complete results for this test case.

The following tests pertain to the four sets of ontologies of the OAEI initiative. In the alignment of the ontologies in the first OAEI set (weapons), the DSI method yielded slightly higher precision and recall values than both the $S S C$ and the Similarity Flooding methods as shown in Table 4.

All four methods yielded the same results for recall and precision in the alignment of the second OAEI set (people and pets) as shown in Table 5. This is an indication that the locality of all the concepts in the ontologies of the second set are irrelevant in distinguishing their identity. 
The SSC method yielded better recall and precision results than the Similarity Flooding algorithm, which in turn yielded better results the the DSI method when aligning the third OAEI set (computer networks) as shown in Table 6. Finally, as shown in Table 7, in the alignment of the fourth OAEI set (Russia), the DSI method yielded the highest results for precision and recall than either the $S S C$ method or the Similarity Flooding algorithm.

The differences found in the recall and precision values for a given method when applied across different test cases are mainly due to the characteristics of the ontologies. For example, in the first OAEI set (weapons) and the second OAEI set (people and pets), the relations between the concepts, their parents, and their siblings do not contribute to refining the base similarity results. However, the relationships between the concepts and their siblings added value in refining the base similarity results when aligning the third OAEI set (computer networks). The relationships between the concepts and their parents added value in refining the results when aligning the fourth OAEI set (Russia). Therefore, the selection of an appropriate matching method should be done after a preliminary examination of the concepts in the ontologies and how they relate to each other. A methodology on how to select an appropriate matching method for a specific alignment has been proposed, where a domain expert fills out a questionnaire about the nature of the ontologies to be aligned (Mochol et al., 2006).

\section{$7 \quad$ Precision and Recall Optimization}

One feature of our system is the ability to specify a similarity threshold when using the automatic methods in our first layer of mapping as discussed in Section 5.1. This feature can help the user in controlling the precision and the recall values during the alignment process. In general, selecting a relatively high similarity threshold value reduces the number of discovered relations because only relations that have a high degree of confidence detected by their similarity measures are considered in the final alignment results. As a result, precision is more likely to increase because it is calculated by dividing the number of valid discovered relations by the number of discovered relations. Recall, on the other hand, is more likely to decrease or stay stable because it is calculated by dividing the number of valid discovered relations by the number of expected valid relations, which is always the same regardless of the similarity threshold. The reason for a potential decrease in recall when the similarity threshold is set high, is the possibility of excluding valid relations that yielded similarity measures below the set similarity threshold. These relations are needed to drive recall higher since 
the total number of discovered relations does not play a role in calculating recall.

When the user selects lower similarity threshold values, the potential of discovering more relations increases. As a result, the number of valid discovered relations generally goes up along with the number of invalid discovered relations, thus generally causing precision to decrease. Recall, on the other hand, generally improves since it only takes into account the number of valid discovered relations (which has increased) and the number of expected valid relations (which remains constant).

We have performed experiments that measure the impact of changing the threshold values on both precision and recall when aligning the four OAEI ontologies. In our experiments, we ran the base similarity method with five threshold values $(20 \%, 40 \%, 60 \%, 80 \%$, and $90 \%)$ when aligning the four ontologies. We also repeated the same experiment with both our DSI and SSC methods. All the three methods exhibited the same behavior on precision and recall, as far as the impact of similarly threshold is concerned. The final results of using the three methods in our experiments were consolidated by averaging the recall and precision values for each of the five aforementioned similarity threshold values.

Figure 8 shows how the precision and recall values change with different similarity thresholds in the alignment of the ontologies for weapons. In the figure, we notice a very slight decrease of the recall and a very slight increase of the precision as the similarity threshold increase.

\section{[ Figure 8 to be placed about here. ]}

In aligning the ontologies for people and pets, we notice an increase in precision and a very slight decrease in recall as the similarity threshold increases, as shown in Figure 9.

\section{[ Figure 9 to be placed about here. ]}

In aligning the computer networks ontologies, we notice a sharp increase in precision when the threshold is set higher than $40 \%$, which is accompanied with a sharp decrease in recall as shown in Figure 10.

\section{[ Figure 10 to be placed about here. ]}

Precision reached its maximum value at threshold values of $80 \%$ and above while recall continued to decrease. Similar behavior is also noticed when aligning the ontologies on Russia as shown in Figure 11. 


\section{[ Figure 11 to be placed about here. ]}

In conclusion, our approach gives flexibility to the user to steer the automatic alignment in favor of optimizing recall or precision. The choice is dependent on the purpose of the alignment process. For example, if the alignment results contribute to an information retrieval system that aims to find the maximum number of possible correspondences regardless of their correctness, then recall can be optimized. If the alignment results are used in a more rigid environment where correspondences are expected to be as much as possible correct, then precision must be optimized.

\section{Ontology Alignment Campaign}

To further validate our approach, we competed in the 2007 Ontology Alignment Campaign (Euzenat et al., 2007) with our DSI method. We participated in the biomedical anatomy track. The biomedical anatomy track is considered a "blind test" in that the results of the alignment, which are contained in a reference alignment document, are hidden from the participants. The reference document contained only equivalence correspondences between concepts of the ontologies. The first ontology in this track described the mouse adult anatomy published by the Mouse Gene Expression Database Project. The second ontology described the human anatomy published by the National Cancer Institute. The first ontology contained 2744 concepts whereas the second ontology contained 3304 concepts. In this track, our system competed against six other systems, which, like ours, can match ontologies in any domain. The quality of the alignments was determined based on the obtained precision (number of discovered valid relations by the total number of discovered relations), recall (number of discovered valid relations to the total number of valid relations), and F-measure (two times the product of precision and recall by their sum).

This track included three test cases, whose aim was respectively to get optimal values for Fmeasure, precision, and recall. In the first test case, our system came in third with an F-measure value of $59.4 \%$ (average was $52.6 \%$ for all the competing systems), a precision value of $55.8 \%$, and a recall value of $63.5 \%$. The F-measure reflects a balance between both precision and recall. In order to obtain a reasonable F-measure value, we set the similarity threshold to $60 \%$. This value was selected moderately (somewhere between 0\% and 100\%) in order to prohibit the establishment of any weak correspondences to optimize recall but so as to allow for stronger correspondences to be established that would optimize precision. We note that we had just one opportunity to run each test case, therefore we could not test different similarity threshold values. 
In the second test case (which aims to optimize precision) our system came in fourth place with a precision value of $93 \%$ (average $86.3 \%$ ). The optimization of precision was achieved by increasing the similarity threshold to $90 \%$ in order to only allow the correspondences with a high degree of confidence to be established. This caused the recall measure to drop sharply to $28.6 \%$. Finally, in the third test case (which aims to optimize recall) the similarity threshold was decreased to $40 \%$ in order to maximize the number of correct correspondences. With this threshold, our system came in third place with a recall value of $65.1 \%$ (average $62.3 \%$ ). The precision for this test case dropped, as expected, to $42.2 \%$. Table 8 shows the complete results of our system and of the other systems that competed in the alignment competition. Figure 12 shows the same results in a chart view.

\section{[ Figure 12 to be placed about here. ]}

We note that we competed for the first time, while several of the other teams have competed in previous years. It has been observed that participation recurrence leads to increasingly better outcomes (Euzenat et al., 2007).

\section{$9 \quad$ Performance Tuning}

In our current approach, all the concepts in the source ontologies are compared to all the concepts in the target ontologies. To tune performance, we use a greedy approach that is more selective in terms of the elements that get compared and can substantially reduce the number of such comparisons.

In our performance tuning approach, we start by comparing the immediate children of the root of the source ontology to the immediate children of the root of the target ontology using the base similarity algorithm. For example, looking at Figure 13, we compare concept $A$ in the source ontology $S$ to concepts $A^{\prime}, B^{\prime}$, and $C^{\prime}$ in the target ontology $T$. Likewise, we compare the other children of $S(B$ and $C$ ) to all the children of $T$.

\section{[ Figure 13 to be placed about here. ]}

After collecting the results of all the comparisons, the best possible match for each of the children of $S$ is determined. Following this, the algorithm proceeds by comparing only the subtrees of the matching concepts using one of our methods (base similarity, DSI, or $S S C$ ). For example, if $A$ in $S$ is matched with $A^{\prime}$ in $T$, only the subtree of $A$ will be compared to the subtree of $A^{\prime}$. This will reduce the number of comparisons significantly and therefore will yield better performance for our algorithm. 
Without our performance tuning approach, the asymptotic time complexity of the $S S C$ and $D S I$ methods is proportional to the product of the number of nodes in each hierarchy, $(|S| \cdot|T|)$ and the time to execute the base similarity method. The complexity of the DSI method has an additional factor that accounts for the minimum of the depths of the two hierarchies. As usual, in this kind of analysis, one disregards the constant factors, which may affect in practice the results obtained (Goodrich and Tamassia, 2002). Also, we would like to point out that asymptotic analysis "makes only sense" when the size of the problems is large.

When adopting our performance tuning approach, the asymptotic time complexity remains the same for general hierarchies. However, in the case that the number of children of each node is bounded by a constant, the asymptotic time complexity is significantly reduced since the product of the number of nodes $(|S| \cdot|T|)$ is replaced by their sum $(|S|+|T|)$. Therefore, in this case, performance tuning reduces from quadratic to linear the dependency of the time complexity on the size of the hierarchies.

The possibility of compromising the quality of the alignment results is expected to increase with performance tuning, because of of a possible reduction in recall and precision values. Figure 14 shows the performance enhancement when applying the performance tuning method for the four OAEI test cases with the base similarity method.

\section{[ Figure 14 to be placed about here. ]}

On average, time was reduced by about $31.2 \%$. The average reduction in the precision values was less than $1 \%$ as shown in Figure 15.

\section{[ Figure 15 to be placed about here. ]}

Looking at the figure, the precision did not change when aligning the ontologies for weapons and for Russia and decreased when aligning the ontologies for people and pets and increased in the case of aligning the ontology for computer networks. The reason for precision increase in some cases is a significant reduction in the number of discovered relations with a small decrease in the number of valid discovered relations. Since precision is calculated as the number of valid discovered relations divided by the number of discovered relations, it can go both ways, either increasing or decreasing. On the other hand, the average recall for the four tests was reduced by around $11 \%$ after tuning the performance as seen in Figure 16.

[ Figure 16 to be placed about here. ] 
Applying the DSI method on the four test cases saved on average $30 \%$ of runtime after performance tuning as seen in Figure 17.

\section{[ Figure 17 to be placed about here. ]}

As a result, the average precision was reduced by around $3 \%$ as shown in Figure 18,

[ Figure 18 to be placed about here. ]

and the average recall was reduced by around $12 \%$ as shown in Figure 19 .

\section{[ Figure 19 to be placed about here. ]}

Applying the $S S C$ method on the four test cases saved on average $31 \%$ of runtime after performance tuning as shown in Figure 20.

\section{[ Figure 20 to be placed about here. ]}

As a result, the average precision was reduced by around $3 \%$ as shown in Figure 21, and the average recall was reduced by around $11 \%$ as seen in Figure 22 .

\section{[ Figure 21 to be placed about here. ]}

\section{[ Figure 22 to be placed about here. ]}

The best result, however, happened for the largest ontologies, the biomedical ontologies of the OAEI 2007 campaign. When tuning the performance for these ontologies, the runtime was reduced from 30 minutes to around 9 minutes with a small impact on both recall and precision (which were reduced by approximately $3 \%$ ). With this new runtime, our system would have taken first place in performance, instead of third place.

\section{Conclusions}

Our motivation to look at automatic ontology alignment, and especially at structured-based methods, stems from the need to integrate heterogeneous data across different geographic areas. The two methods that we propose, the Descendant's Similarity Inheritance (DSI) method and the Sibling's Similarity Contribution (SSC) method use respectively the information associated with the ancestors and with the siblings of each concept, which are used in conjunction with a conceptbased method, the base similarity method. We have implemented our methods and evaluated them 
on several ontology sets. We also established a comparison with the Similarity Flooding algorithm (Melnik et al., 2002). To further validate our approach, we entered the OAEI competition where our methods faired well. We studied the influence of the choice of a threshold value for the base similarity method on precision and recall and optimized the implementation of our methods for performance. Experimental results showed that we obtained significant performance gains at little cost to both precision and recall.

Much work remains to be accomplished in the general area of ontology alignment and in particular in the area of geospatial ontology alignment.

A general issue is the determination of which methods to use depending on the ontologies involved and on their particular topologies. For example, the fact that the most effective method is not always the same and that sometimes all the four methods have similar results shows that: (1) the best method depends on the topology of the ontology graph and (2) for certain topologies, structure-based methods do not play an important role. Both of these conclusions have been arrived at by others (Mochol et al., 2006) and they further justify our multi-layered approach where several techniques can be used and combined. As more criteria gets added, the greater is the the number of possibilities built (Tang et al., 2006) and the complexity in merging all the results (Silva et al., 2005). Thus, it is important that optimization occurs as soon as possible and that the combination methods are also efficient and, when iterative, may discard safely solutions that are not optimal as soon as possible.

We have been considering hierarchical classification schemes. However, if we move to other applications or to a richer semantic model, then directed acyclic graphs or even cyclic graphs need to be considered (Euzenat and Valtchev, 2004).

Initiatives like the OAEI need to incorporate ontology sets for geospatial applications. Our experience with geospatial applications and domain experts has provided us with insight into the problem of developing such sets. First of all, the ontologies must be comparable and good candidates for matching. Second, both ontologies need to refer to the same aspects of reality and must be well described and structured. Third, building a reference alignment between ontologies for testing purposes should be attempted with care: involving more than one domain expert with this task can significantly improve the precision of the reference alignment - the basis for measuring the effectiveness of an automatic approach, which the domain experts can rely upon. 


\section{Acknowledgments}

This paper revises and extends the previous paper by the authors: William Sunna and Isabel F. Cruz. "Structure-based Methods to Enhance Geospatial Ontology Alignment," Second International Conference on GeoSpatial Semantics (GeoS), Mexico City, LNCS 4853, pp. 82-97, Springer, 2007. This research was supported in part by the National Science Foundation under Awards ITR IIS-0326284, IIS-0513553, and IIS-0812258.

We would like to thank Sarang Kapadia for his help with the implementation. We would also

like to thank Nancy Wiegand and Steve Ventura from the Land Information \& Computer Graphics Facility at the University of Wisconsin-Madison for discussions on geospatial problems and for facilitating our meetings with domain experts. 


\section{References}

Sonia Bergamaschi, Silvana Castano, and Maurizio Vincini. Semantic Integration of Semistructured and Structured Data Sources. SIGMOD Record, 28(1):54-59, 1999.

Silvana Castano, Valeria De Antonellis, and Sabrina De Capitani di Vimercati. Global Viewing of Heterogeneous Data Sources. IEEE Transactions on Knowledge and Data Engineering, 13(2): 277-297, 2001.

Lewis M. Cowardin, Virginia Carter, Francis C. Golet, and Edward T. LaRoe. Classification of Wetlands and Deepwater Habitats of the United States. U.S. Department of the Interior, Fish and Wildlife Service, Washington, D.C. Jamestown, ND: Northern Prairie Wildlife Research Center Online. http://www.npwrc.usgs.gov/resource/wetlands/classwet/index.htm (Version 04DEC1998), 1979.

Isabel F. Cruz and Huiyong Xiao. The Role of Ontologies in Data Integration. Journal of Engineering Intelligent Systems, 13(4):245-252, December 2005.

Isabel F. Cruz, Afsheen Rajendran, William Sunna, and Nancy Wiegand. Handling Semantic Heterogeneities using Declarative Agreements. In ACM Symposium on Advances in Geographic Information Systems (ACM GIS), pages 168-174, 2002.

Isabel F. Cruz, William Sunna, and Anjli Chaudhry. Semi-Automatic Ontology Alignment for Geospatial Data Integration. In International Conference on Geographic Information Science (GIScience), volume 3234 of Lecture Notes in Computer Science, pages 51-66. Springer, 2004.

Isabel F. Cruz, William Sunna, and Kalyan Ayloo. Concept Level Matching of Geospatial Ontologies. In GISPlanet Second Conference and Exhibition on Geographic Information, 2005.

Isabel F. Cruz, William Sunna, Nalin Makar, and Sujan Bathala. A Visual Tool for Ontology Alignment to Enable Geospatial Interoperability. Journal of Visual Languages and Computing, $18(3): 230-254,2007$.

Klaas Dellschaft and Steffen Staab. On How to Perform a Gold Standard Based Evaluation of Ontology Learning. In Isabel F. Cruz, Stefan Decker, Dean Allemang, Chris Preist, Daniel Schwabe, Peter Mika, Michael Uschold, and Lora Aroyo, editors, International Semantic Web Conference (ISWC), volume 4273 of Lecture Notes in Computer Science, pages 228-241, 2006. 
J. Dini, G. Gowan, and P. Goodman. South African National Wetland Inventory, Proposed Wetland Classification System for South Africa. http://www.ngo.grida.no/soesa/nsoer/resource/wetland/inventory_classif.htm, 1998.

AnHai Doan, Jayant Madhavan, Pedro Domingos, and Alon Y. Halevy. Learning to Map between Ontologies on the Semantic Web. In International World Wide Web Conference $(W W W)$, pages 662-673, 2002.

Jérôme Euzenat and Petko Valtchev. Similarity-Based Ontology Alignment in OWL-Lite. In European Conference on Artificial Intelligence (ECAI), pages 333-337. IOS Press, 2004.

Jérôme Euzenat, Philippe Guégan, and Petko Valtchev. OLA in the OAEI 2005 Alignment Contest. In K-CAP Workshop on Integrating Ontologies, volume 156 of CEUR Workshop Proceedings, 2005 .

Jérôme Euzenat, Christian Meilicke, Pavel Shvaiko, Heiner Stuckenschmidt, Ondrej Šváb, Vojtech Svátek, Willem Robert van Hage, and Mikalai Yatskevich. First Results of the Ontology Evaluation Initiative 2007. In ISWC International Workshop on Ontology Matching. CEUR-WS, 2007.

Davide Fossati, Gabriele Ghidoni, Barbara Di Eugenio, Isabel F. Cruz, Huiyong Xiao, and Rajen Subba. The Problem of Ontology Alignment on the Web: a First Report. In Web as Corpus Workshop (associated with the Conference of the European Chapter of the ACL), 2006.

Michael T. Goodrich and Roberto Tamassia. Algorithm Design: Foundations, Analysis and Internet Examples. John Wiley \& Sons, New York, NY, 2002.

David A. Hull. Stemming Algorithms: A Case Study for Detailed Evaluation. Journal of the American Society of Information Science, 47(1):70-84, 1996.

Ryutaro Ichise, Hideaki Takeda, and Shinichi Honiden. Rule Induction for Concept Hierarchy Alignment. In IJCAI Workshop on Ontologies and Information Sharing, 2001.

Maurizio Lenzerini. Data Integration: A Theoretical Perspective. In ACM SIGMOD-SIGACTSIGART Symposium on Principles of Database Systems (PODS), pages 233-246, 2002.

Dekang Lin. An Information-Theoretic Definition of Similarity. In International Conference on Machine Learning (ICML), pages 296-304. Morgan Kaufmann, 1998. 
Alexander Maedche and Steffen Staab. Measuring Similarity between Ontologies. In International Conference on Knowledge Engineering and Knowledge Management (EKAW), volume 2473 of Lecture Notes in Computer Science, pages 251-263. Springer, 2002.

Sergey Melnik, Hector Garcia-Molina, and Erhard Rahm. Similarity Flooding: A Versatile Graph Matching Algorithm and its Application to Schema Matching. In IEEE International Conference on Data Engineering (ICDE), pages 117-128, 2002.

Malgorzata Mochol, Anja Jentzsch, and Jérôme Euzenat. Applying an Analytic Method for Matching Approach Selection. In International Workshop on Ontology Matching (OM) collocated with the International Semantic Web Conference (ISWC), 2006.

Natalya Fridman Noy and Mark A. Musen. Anchor-PROMPT: Using Non-local Context for Semantic Matching. In IJCAI Workshop on Ontologies and Information Sharing, 2001.

Luigi Palopoli, Domenico Saccà, and Domenico Ursino. An Automatic Techniques for Detecting Type Conflicts in Database Schemes. In International Conference on Information and Knowledge Management (CIKM), pages 306-313, 1998.

Philip Resnik. Using Information Content to Evaluate Semantic Similarity in a Taxonomy. In International Joint Conference on Artificial Intelligence (IJCAI), pages 448-453, 1995.

M. Andrea Rodríguez and Max J. Egenhofer. Determining Semantic Similarity among Entity Classes from Different Ontologies. IEEE Transactions on Knowledge and Data Engineering, 15 (2):442-456, 2003.

Pavel Shvaiko and Jérôme Euzenat. A Survey of Schema-Based Matching Approaches. In Journal on Data Semantics IV, volume 3730 of Lecture Notes in Computer Science, pages 146-171. Springer, 2005.

Nuno Silva, Paulo Maio, and João Rocha. An Approach to Ontology Mapping Negotiation. In K-CAP Workshop on Integrating Ontologies, volume 156 of CEUR Workshop Proceedings, 2005.

William Sunna and Isabel F. Cruz. Structure-Based Methods to Enhance Geospatial Ontology Alignment. In International Conference on GeoSpatial Semantics (GeoS), volume 4853 of Lecture Notes in Computer Science, pages 82-97. Springer, 2007a. 
William Sunna and Isabel F. Cruz. Using the AgreementMaker to Align Ontologies for the OAEI Campaign 2007. In ISWC International Workshop on Ontology Matching, volume 304. CEURWS, 2007b.

Y. Sure, O. Corcho, J. Euzenat, and T. Hughes. Evaluation of Ontology-based Tools. In International Workshop on Evaluation of Ontology-based Tools (EON). CEUR-WS, 2004.

Jie Tang, Juanzi Li, Bangyong Liang, Xiaotong Huang, Yi Li, and Kehong Wang. Using Bayesian Decision for Ontology Mapping. Journal of Web Semantics, 4(4):243-262, 2006.

Nancy Wiegand, Dan Patterson, Naijun Zhou, Steve Ventura, and Isabel F. Cruz. Querying Heterogeneous Land Use Data: Problems and Potential. In National Conference on Digital Government Research (dg.o), pages 115-121, 2002. 


\begin{tabular}{|l|r|r|r|r|r|}
\hline Characteristics & Wetlands & Weapons & People and pets & Computer networks & Russia \\
\hline Depth & 5 & 6 & 4 & 5 & 5 \\
\hline $\begin{array}{l}\text { Number of concepts in } \\
\text { the source ontology }\end{array}$ & 29 & 153 & 65 & 90 & 86 \\
\hline $\begin{array}{l}\text { Number of concepts in } \\
\text { the target ontology }\end{array}$ & 29 & 213 & 93 & 89 & 87 \\
\hline
\end{tabular}

Table 1: Depth and number of concepts in the ontology sets. 


\begin{tabular}{|l|r|r|r|r|r|}
\hline Algorithm & Wetlands & Weapons & People and pets & Computer networks & Russia \\
\hline Base Similarity & 125 & 1516 & 236 & 391 & 484 \\
\hline DSI & 156 & 3656 & 562 & 579 & 844 \\
\hline SSC & 172 & 4344 & 938 & 719 & 1891 \\
\hline Similarity Flooding & 187 & 2266 & 703 & 906 & 1796 \\
\hline
\end{tabular}

Table 2: Performance results for the base similarity, DSI, SSC, and SF algorithms (Melnik et al., 2002) in milliseconds. 


\begin{tabular}{|l|r|r|r|r|r|}
\hline Algorithm & Total correct relations & Discovered relations & Correct relations & Precision & Recall \\
\hline Base similarity & 54 & 39 & 24 & $61.54 \%$ & $44.44 \%$ \\
\hline DSI & 54 & 39 & 37 & $94.87 \%$ & $68.52 \%$ \\
\hline SSC & 54 & 39 & 29 & $74.36 \%$ & $53.70 \%$ \\
\hline Similarity flooding & 54 & 39 & 36 & $92.31 \%$ & $66.67 \%$ \\
\hline
\end{tabular}

Table 3: Precision and recall for the DSI, SSC, and $S F$ algorithms (wetlands ontology set). 


\begin{tabular}{|l|r|r|r|r|r|}
\hline Algorithm & Total correct relations & Discovered relations & Correct relations & Precision & Recall \\
\hline Base similarity & 73 & 78 & 64 & $82.05 \%$ & $87.67 \%$ \\
\hline DSI & 73 & 78 & 66 & $84.62 \%$ & $90.41 \%$ \\
\hline SSC & 73 & 78 & 65 & $83.33 \%$ & $89.04 \%$ \\
\hline Similarity flooding & 73 & 78 & 65 & $83.33 \%$ & $89.04 \%$ \\
\hline
\end{tabular}

Table 4: Precision and recall for the $D S I, S S C$, and $S F$ algorithms (weapons ontology set). 


\begin{tabular}{|l|r|r|r|r|r|}
\hline Algorithm & Total correct relations & Discovered relations & Correct relations & Precision & Recall \\
\hline Base similarity & 74 & 81 & 49 & $60.49 \%$ & $66.22 \%$ \\
\hline DSI & 74 & 81 & 49 & $60.49 \%$ & $66.22 \%$ \\
\hline SSC & 74 & 81 & 49 & $60.49 \%$ & $66.22 \%$ \\
\hline Similarity flooding & 74 & 81 & 49 & $60.49 \%$ & $66.22 \%$ \\
\hline
\end{tabular}

Table 5: Precision and recall for the DSI, SSC, and SF algorithms (people and pets ontology set). 


\begin{tabular}{|l|r|r|r|r|r|}
\hline Algorithm & Total correct relations & Discovered relations & Correct relations & Precision & Recall \\
\hline Base similarity & 29 & 23 & 17 & $73.91 \%$ & $58.62 \%$ \\
\hline DSI & 29 & 23 & 14 & $60.87 \%$ & $48.28 \%$ \\
\hline SSC & 29 & 23 & 19 & $82.61 \%$ & $65.52 \%$ \\
\hline Similarity flooding & 29 & 23 & 18 & $78.26 \%$ & $62.07 \%$ \\
\hline
\end{tabular}

Table 6: Precision and recall for the DSI, SSC, and SF algorithms (computer and networks ontology set). 


\begin{tabular}{|l|r|r|r|r|r|}
\hline Algorithm & Total correct relations & Discovered relations & Correct relations & Precision & \multicolumn{1}{l|}{ Recall } \\
\hline Base similarity & 117 & 51 & 45 & $88.24 \%$ & $38.46 \%$ \\
\hline DSI & 117 & 51 & 48 & $94.12 \%$ & $41.03 \%$ \\
\hline SSC & 117 & 51 & 46 & $90.20 \%$ & $39.32 \%$ \\
\hline Similarity flooding & 117 & 51 & 46 & $90.20 \%$ & $39.32 \%$ \\
\hline
\end{tabular}

Table 7: Precision and recall for the DSI, SSC, and SF algorithms (Russia ontology set). 


\begin{tabular}{|l|c|c|c|c|c|c|c|}
\hline & \multicolumn{3}{|c|}{$\begin{array}{c}\text { Test Case 1 } \\
\text { (Optimized F-Measure) }\end{array}$} & \multicolumn{2}{c|}{$\begin{array}{c}\text { Test Case II } \\
\text { (Optimized Precision) }\end{array}$} & \multicolumn{2}{c|}{$\begin{array}{c}\text { Test Case III } \\
\text { (0ptimized Recall) }\end{array}$} \\
\hline SYSTEM & Precision & Recall & F-Measure & Precision & Recall & Precision & Recall \\
\hline Falcon-AO & 0.964 & 0.591 & 0.733 & 0.986 & 0.54 & 0.814 & 0.655 \\
\hline TaxoMap & 0.596 & 0.732 & 0.657 & 0.985 & 0.642 & - & - \\
\hline AgreementMaker & 0.558 & 0.635 & 0.594 & 0.93 & 0.286 & 0.424 & 0.651 \\
\hline Prior+ & 0.594 & 0.59 & 0.592 & 0.663 & 0.497 & 0.371 & 0.657 \\
\hline Lily & 0.481 & 0.559 & 0.517 & 0.672 & 0.38 & 0.401 & 0.588 \\
\hline X-SOM & 0.916 & 0.248 & 0.39 & 0.942 & 0.104 & 0.783 & 0.565 \\
\hline DSSim & 0.208 & 0.187 & 0.197 & - & - & - & - \\
\hline
\end{tabular}

Table 8: OAEI 2007 biomedical anatomy track results. 


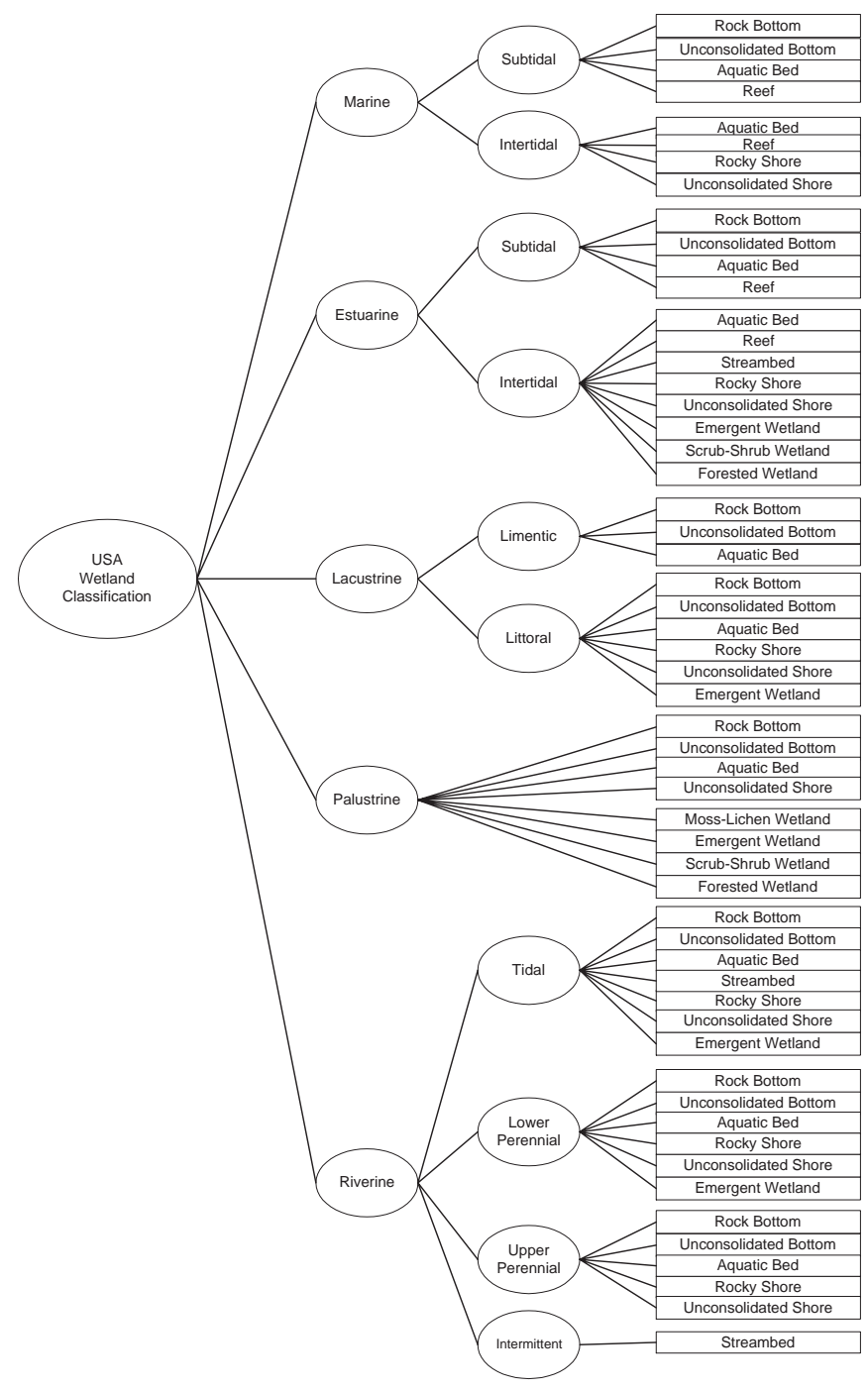

Figure 1: Cowardin wetland ontology (used in the USA) (Cruz et al., 2005). 


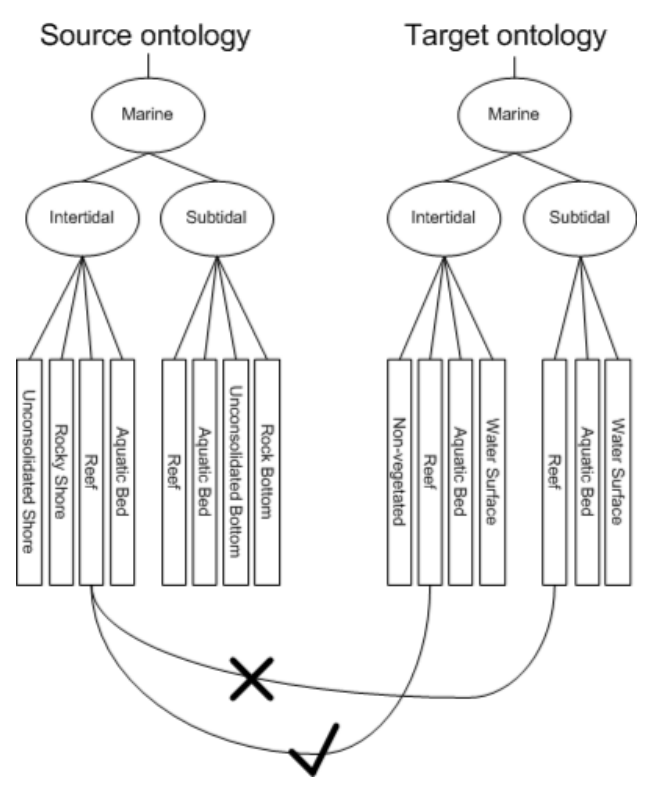

Figure 2: Correct $(\sqrt{ })$ and incorrect $(X)$ mappings between concepts that have the same label. 


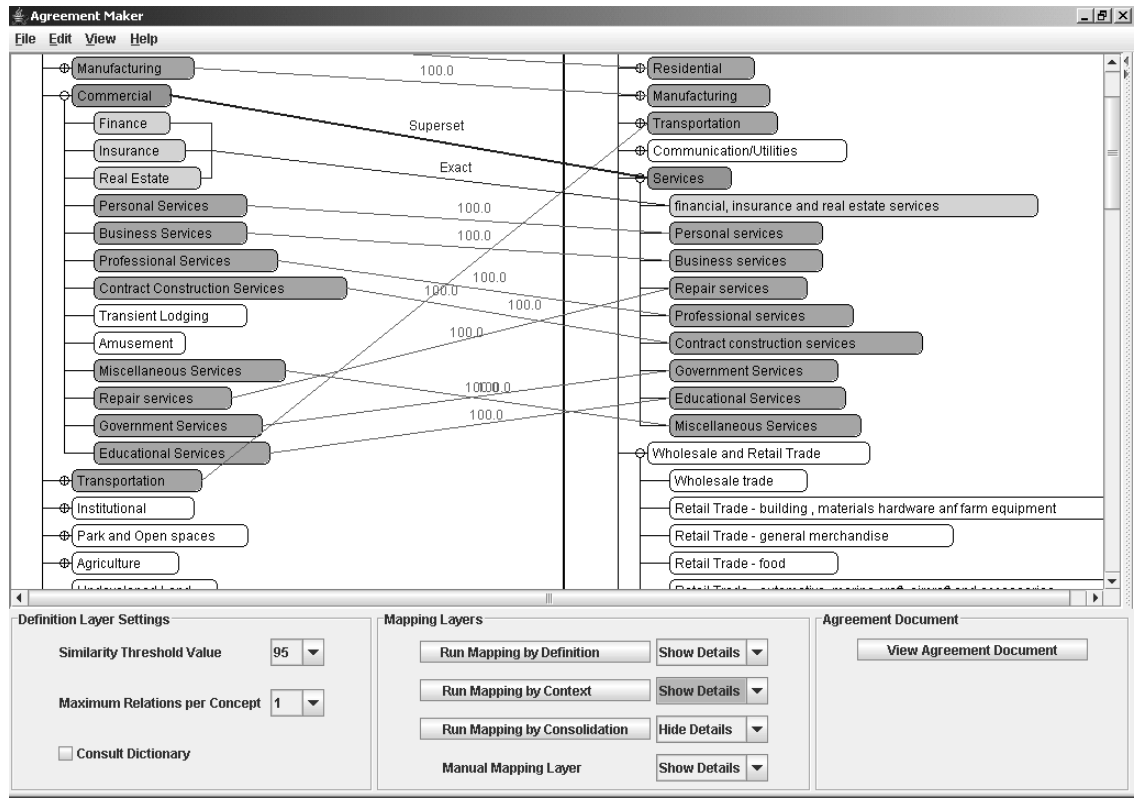

Figure 3: AgreementMaker user interface displaying the results of running three of the mapping layers on land use ontologies. 


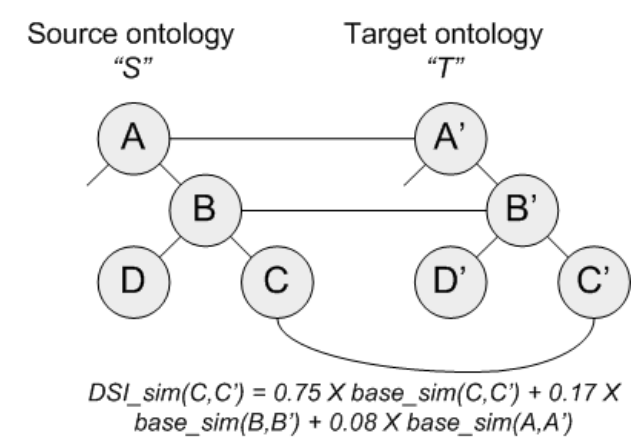

Figure 4: Applying the DSI method to calculate the similarity between $C$ and $C^{\prime}$. 


\section{Source ontology}

"S"

A

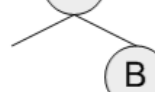

(E) $C$

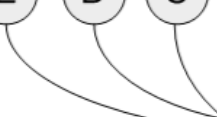

$S S C \operatorname{sim}\left(C, C^{\prime}\right)=0.75 X$ base $\operatorname{sim}\left(C, C^{\prime}\right)+0.25 / 2 X$

(MAX(base_sim $\left(D, D^{\prime}\right)$, base_sim( $\left(D, E^{\prime}\right)$, base_sim $\left.\left(D, F^{\prime}\right)\right)+$

$\operatorname{MAX}\left(\right.$ base_sim $\left(E, D^{\prime}\right)$, base_sim $\left(E, E^{\prime}\right)$, base_sim $\left.\left(E, F^{\prime}\right)\right)$ )

Figure 5: Applying the $S S C$ method to calculate the similarity between $C$ and $C^{\prime}$. 


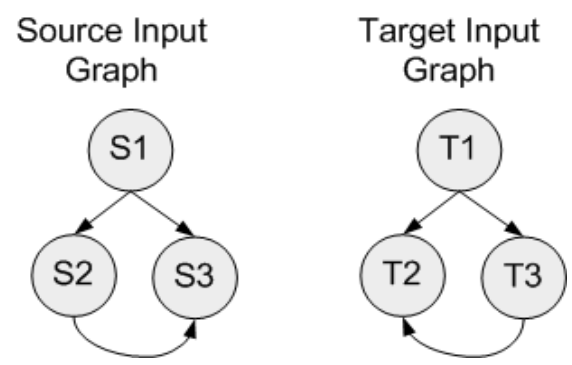

Figure 6: Two input graphs to be aligned by the $S F$ algorithm. 


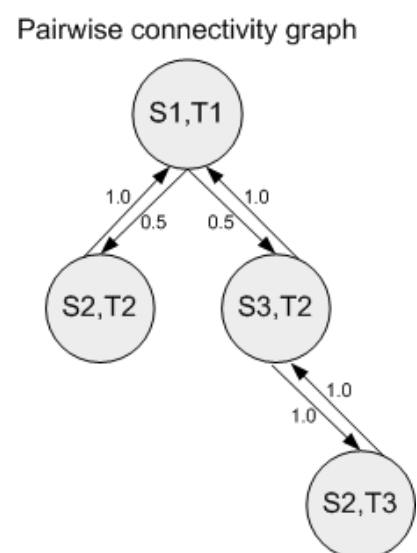

Figure 7: Induced propagation graph showing how similarity is propagated between neighbors. 


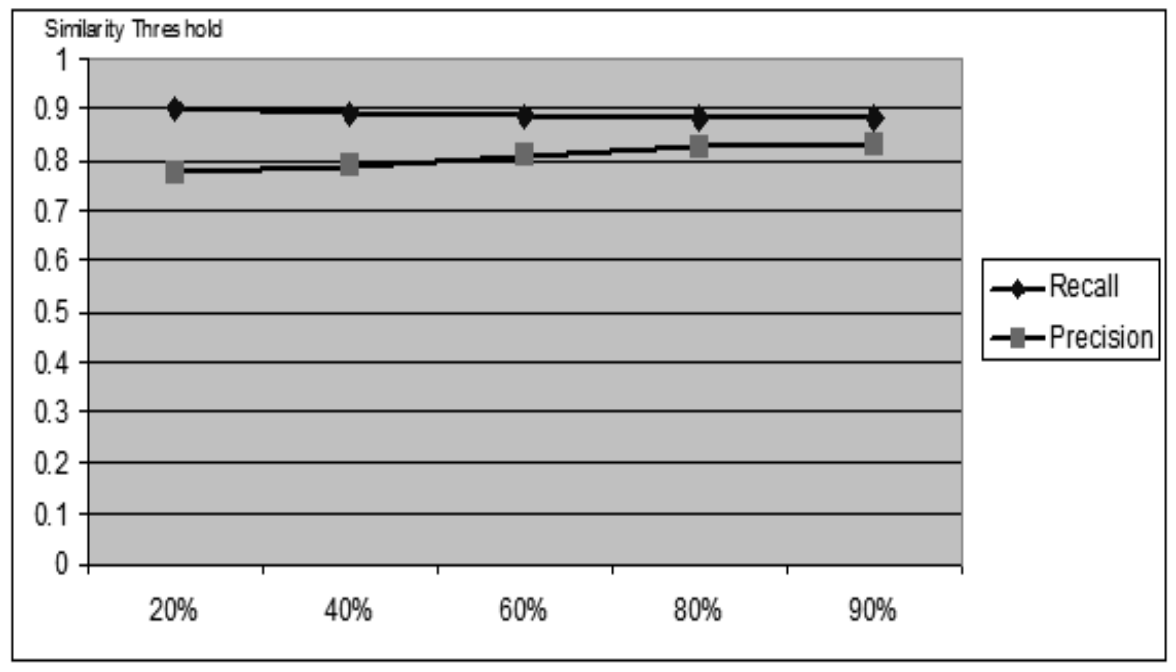

Figure 8: Impact of similarity threshold values on precision and recall (weapons ontology set). 


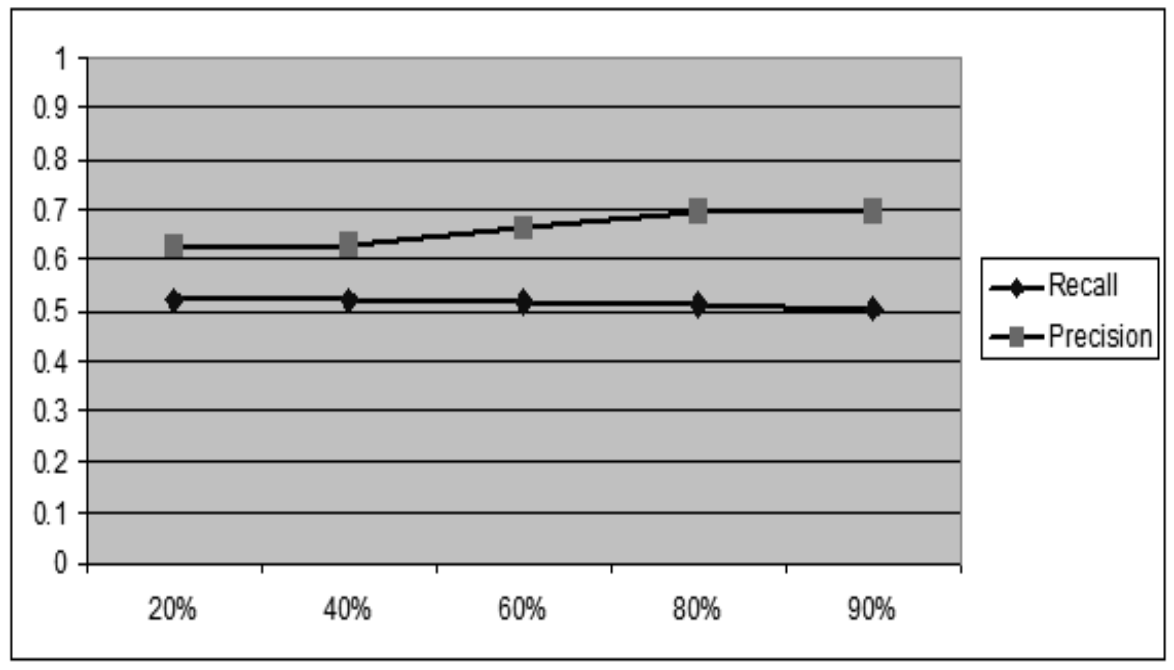

Figure 9: Impact of similarity threshold values on precision and recall (people and pets ontology set). 


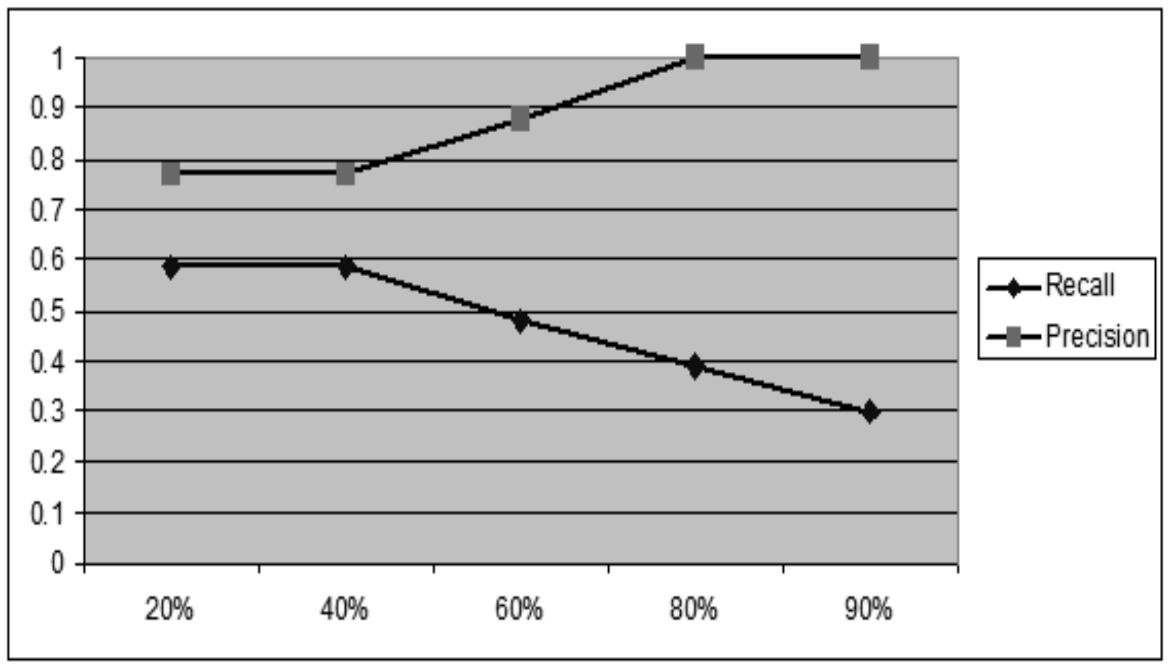

Figure 10: Impact of similarity threshold values on precision and recall (computer networks ontology set). 


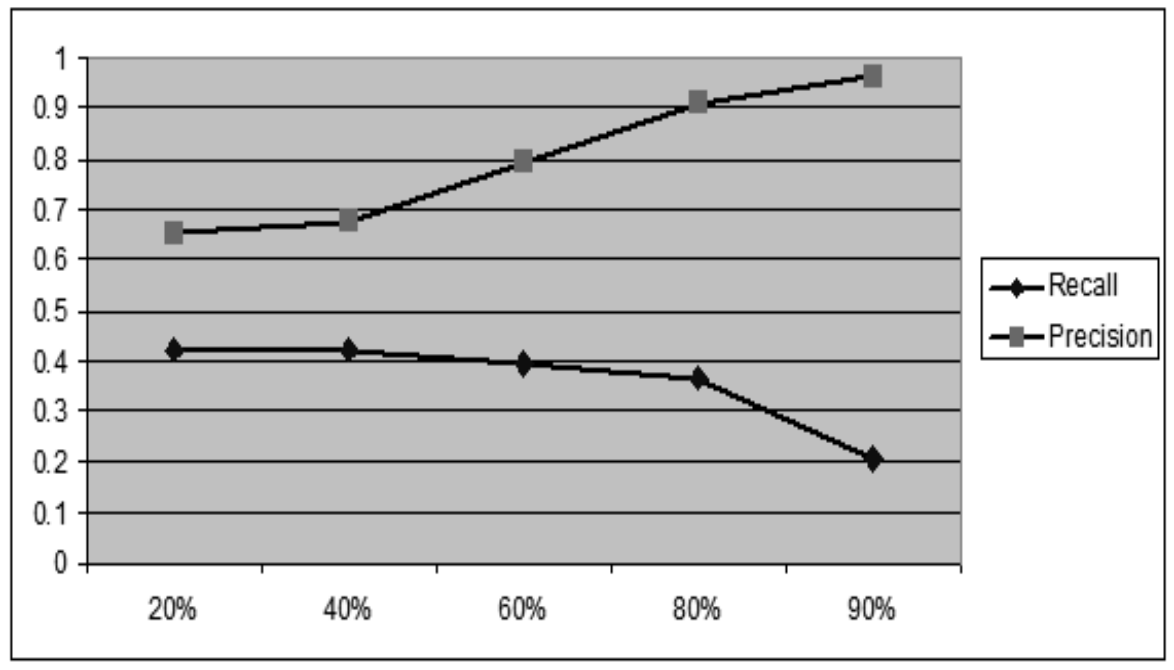

Figure 11: Impact of similarity threshold values on precision and recall (Russia ontology set). 


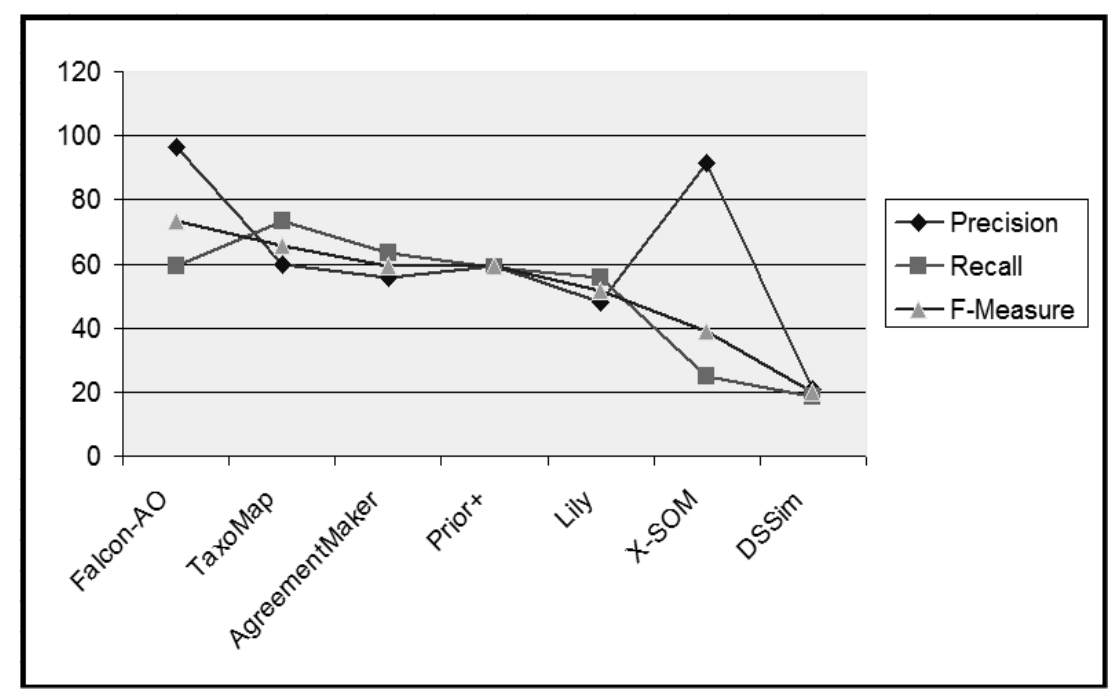

Figure 12: F-measure, precision, and recall for the competing systems in the first test case of the OAEI 2007 anatomical track. 
(s)

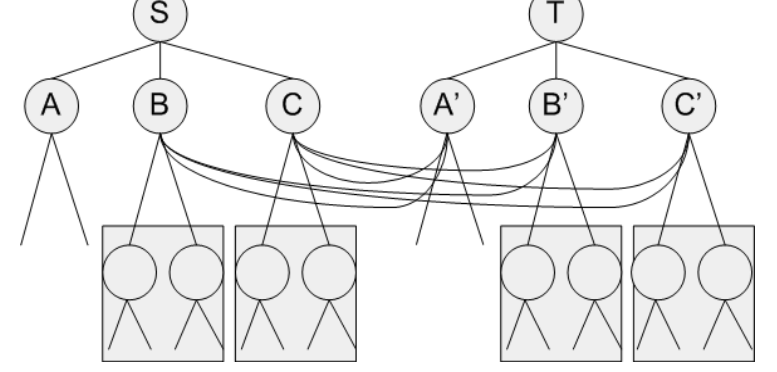

Figure 13: Performance tuning using subtree matching. 


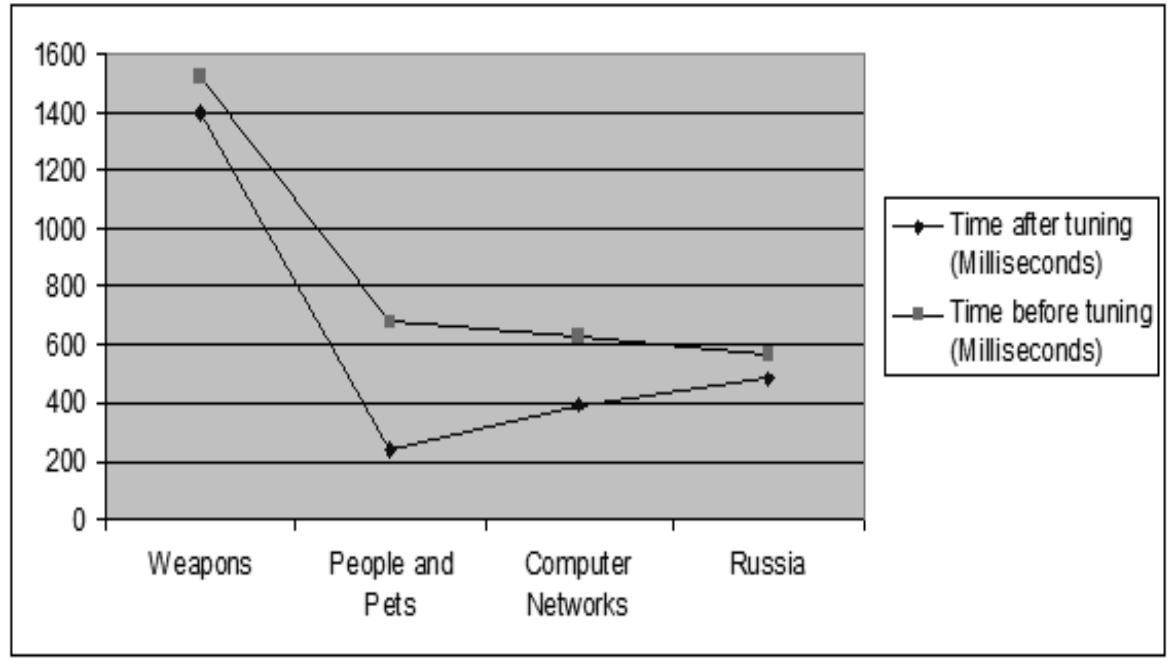

Figure 14: Runtime before and after performance tuning for the base similarity method for the four test cases. 


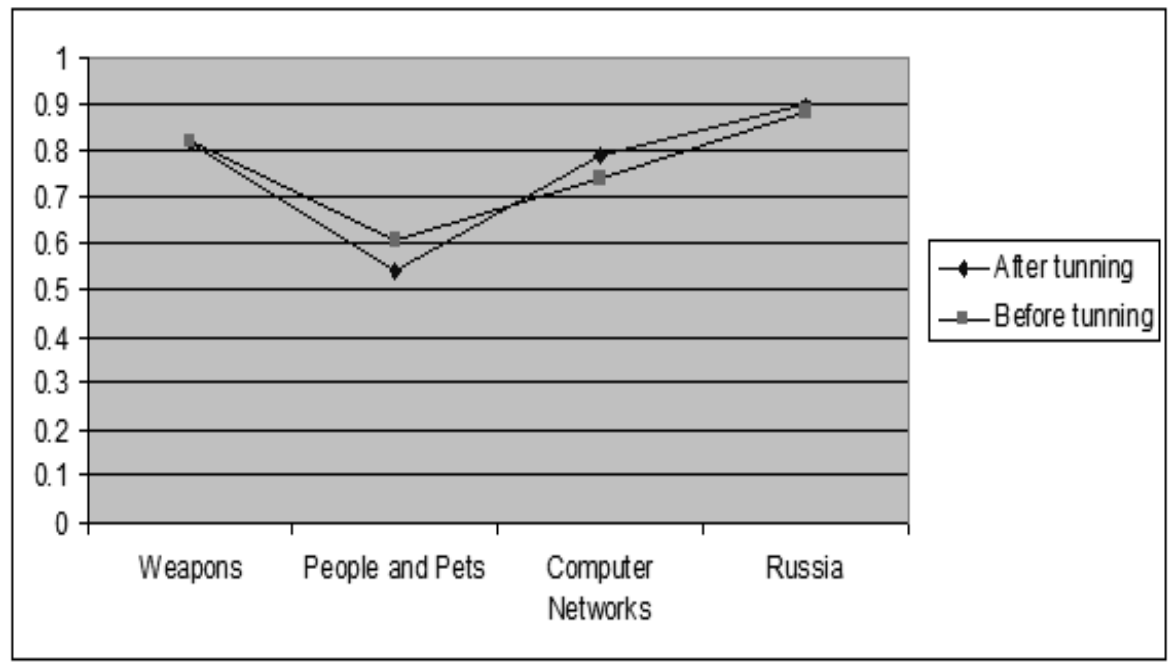

Figure 15: Impact of performance tuning on the base similarity precision values for the four test cases. 


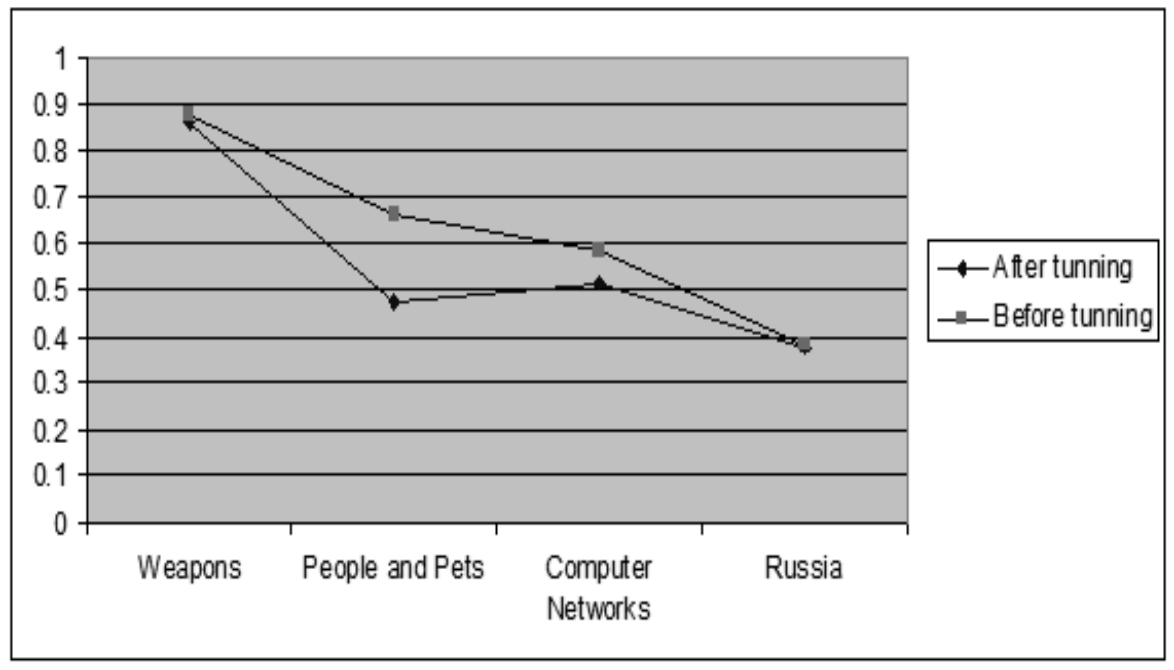

Figure 16: Impact of performance tuning on the base similarity recall values for the four test cases. 


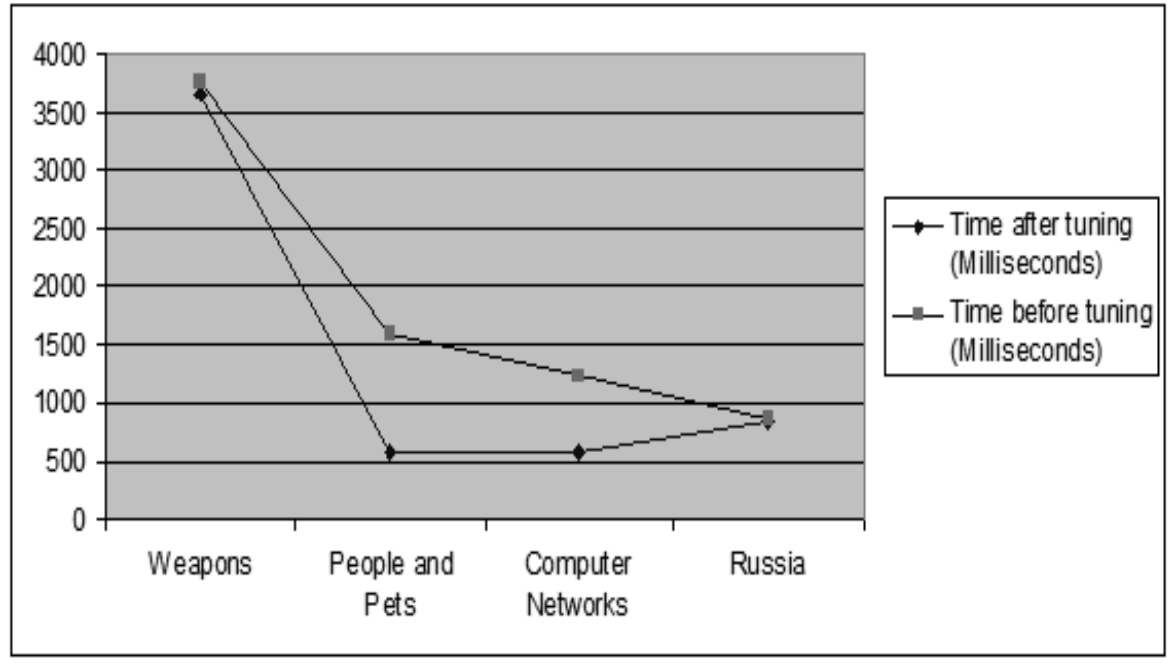

Figure 17: Runtime before and after performance tuning for the $D S I$ for the four test cases. 


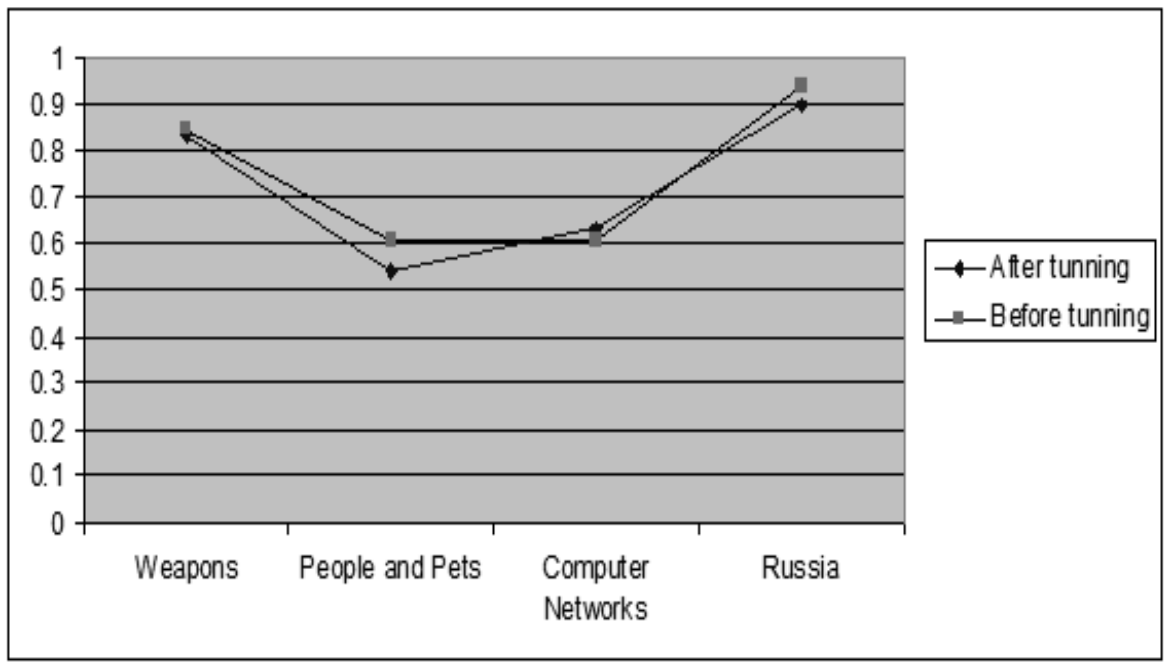

Figure 18: Impact of performance tuning on the $D S I$ precision values for the four test cases. 


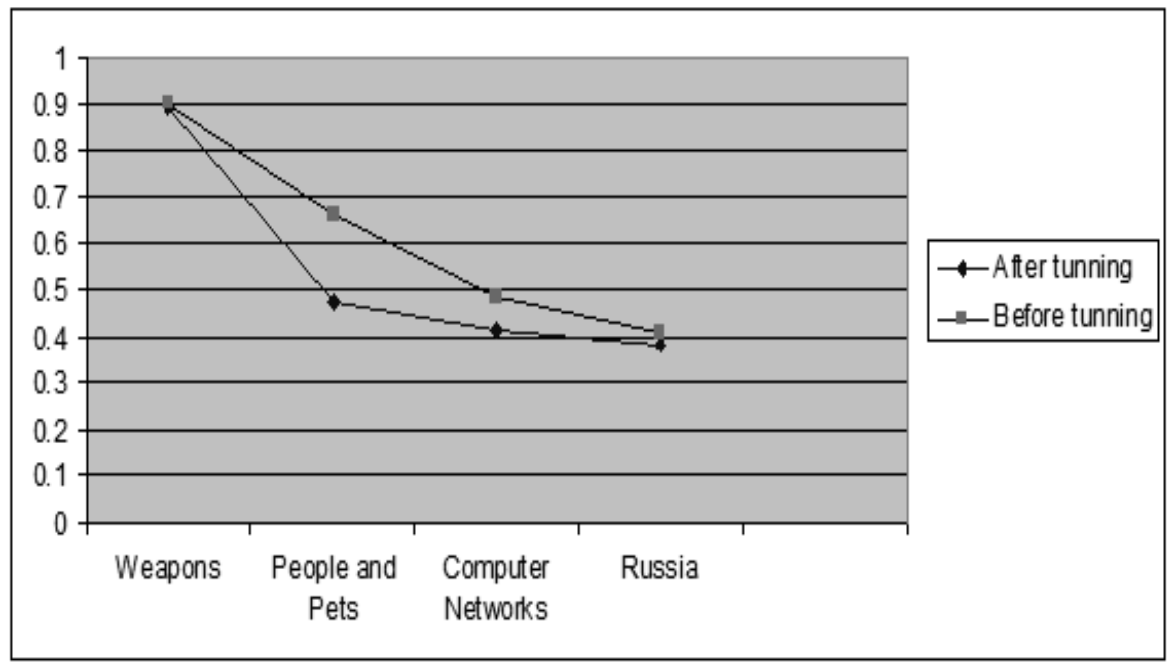

Figure 19: Impact of performance tuning on the $D S I$ recall values for the four test cases. 


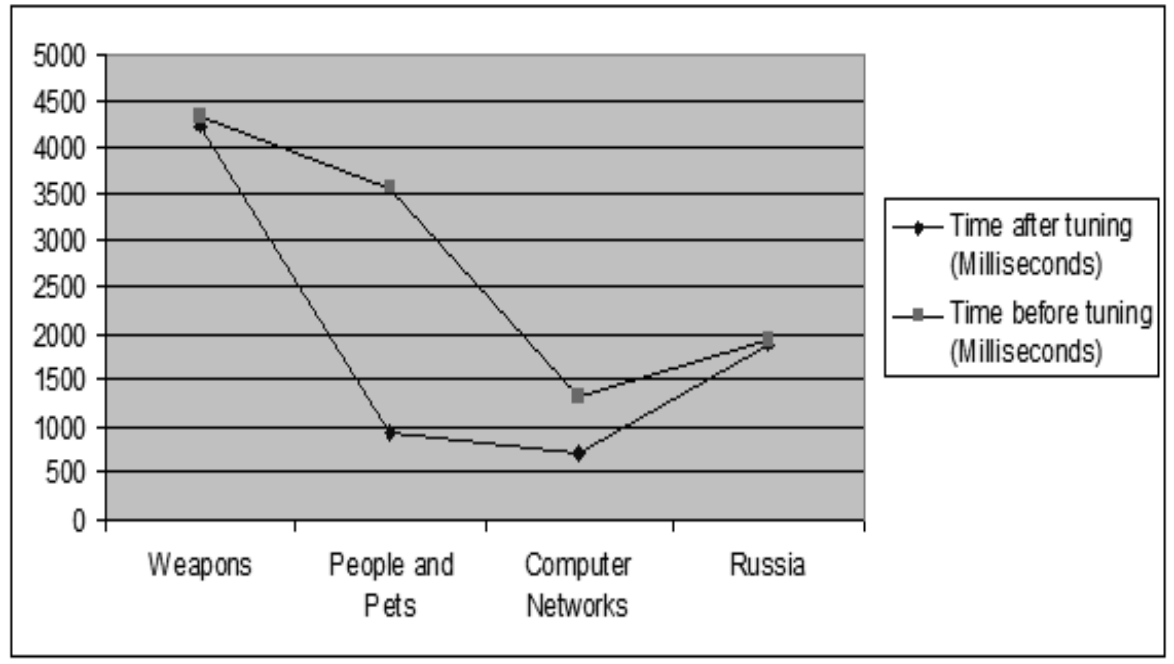

Figure 20: Runtime before and after performance tuning for the $S S C$ for the four test cases. 


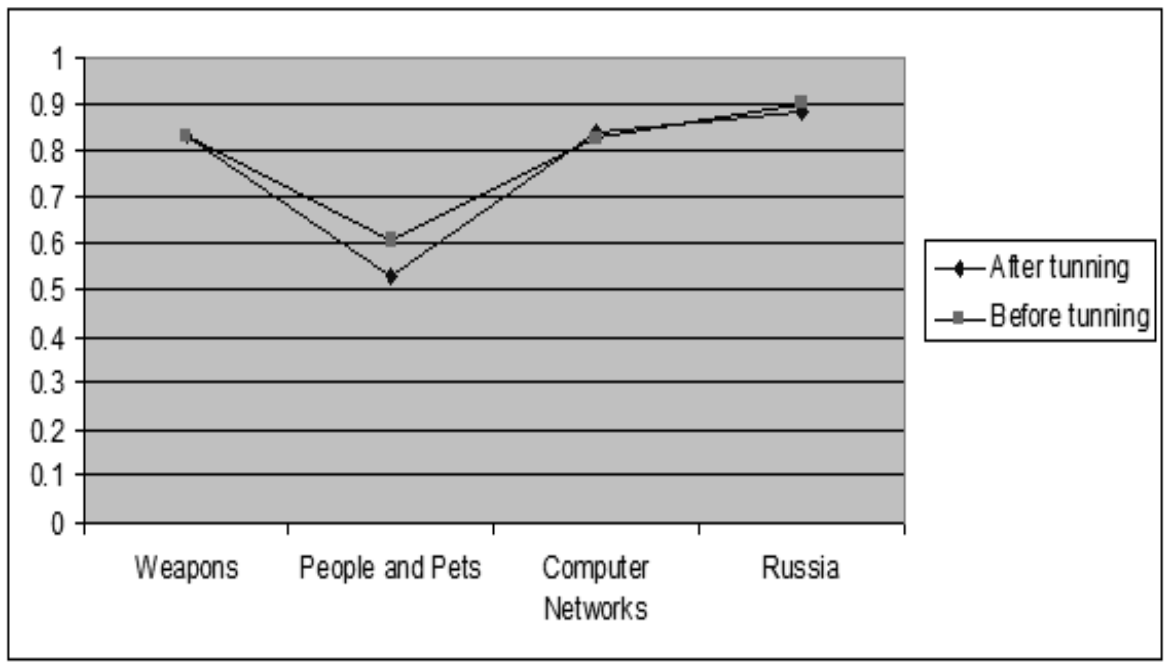

Figure 21: Impact of performance tuning on the $S S C$ precision values for the four test cases. 


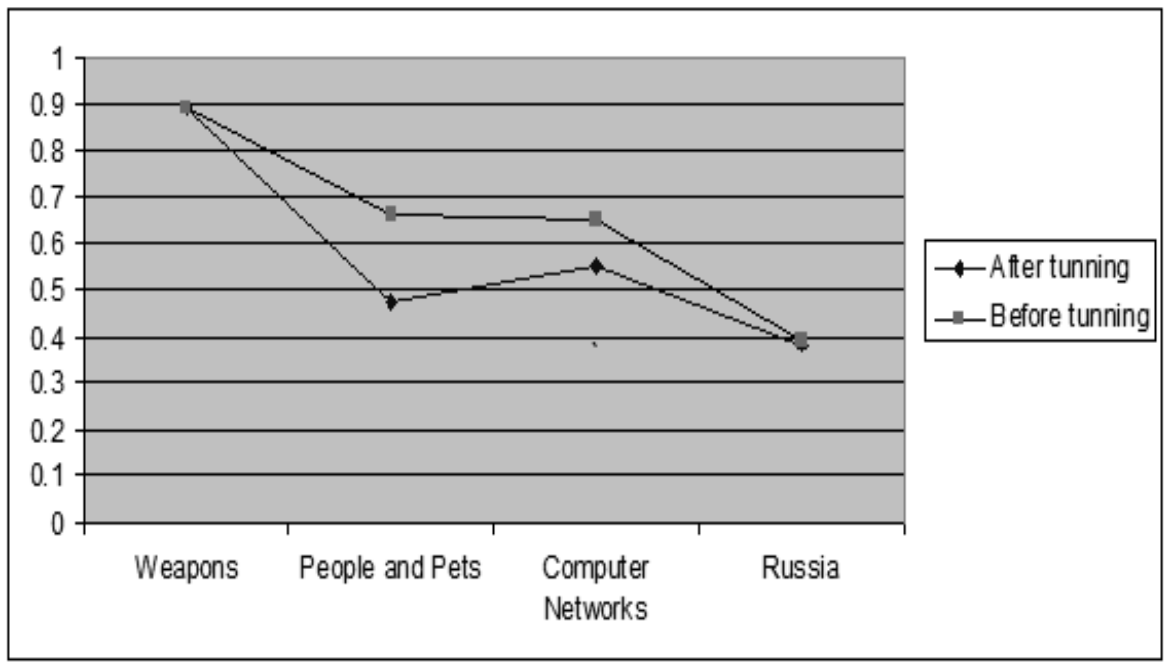

Figure 22: Impact of performance tuning on the $S S C$ recall values for the four test cases. 


\section{List of Tables}

1 Depth and number of concepts in the ontology sets. . . . . . . . . . . 30

2 Performance results for the base similarity, DSI, SSC, and SF algorithms (Melnik et al., 2002) in milliseconds. . . . . . . . . . . . . . . . . . . 31

$3 \quad$ Precision and recall for the DSI, SSC, and SF algorithms (wetlands ontology set). . 32

$4 \quad$ Precision and recall for the $D S I, S S C$, and $S F$ algorithms (weapons ontology set). . . 33

$5 \quad$ Precision and recall for the $D S I, S S C$, and $S F$ algorithms (people and pets ontology

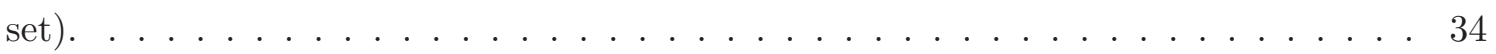

6 Precision and recall for the DSI, SSC, and SF algorithms (computer and networks

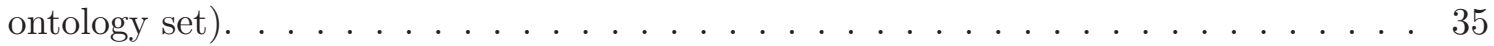

$7 \quad$ Precision and recall for the $D S I, S S C$, and $S F$ algorithms (Russia ontology set). . . 36

$8 \quad$ OAEI 2007 biomedical anatomy track results. . . . . . . . . . . . . . . 37 


\section{List of Figures}

1 Cowardin wetland ontology (used in the USA) (Cruz et al., 2005). . . . . . . . . 38

2 Correct $(\sqrt{ })$ and incorrect $(X)$ mappings between concepts that have the same label. 39

3 AgreementMaker user interface displaying the results of running three of the mapping layers on land use ontologies. . . . . . . . . . . . . . . . . . . 40

4 Applying the $D S I$ method to calculate the similarity between $C$ and $C^{\prime} \ldots \ldots 4$

5 Applying the $S S C$ method to calculate the similarity between $C$ and $C^{\prime} \ldots \ldots 42$

6 Two input graphs to be aligned by the $S F$ algorithm. . . . . . . . . . . . . . . 43

$7 \quad$ Induced propagation graph showing how similarity is propagated between neighbors. 44

8 Impact of similarity threshold values on precision and recall (weapons ontology set). 45

9 Impact of similarity threshold values on precision and recall (people and pets ontol-

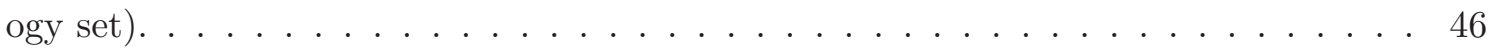

10 Impact of similarity threshold values on precision and recall (computer networks

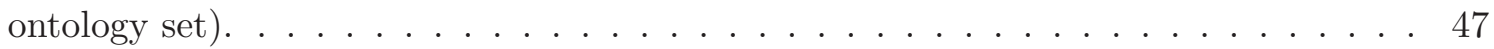

11 Impact of similarity threshold values on precision and recall (Russia ontology set). . 48

12 F-measure, precision, and recall for the competing systems in the first test case of the OAEI 2007 anatomical track . . . . . . . . . . . . . . . . . . . . 49

13 Performance tuning using subtree matching. . . . . . . . . . . . . . . . 50

14 Runtime before and after performance tuning for the base similarity method for the four test cases. . . . . . . . . . . . . . . . . . . . . 51

15 Impact of performance tuning on the base similarity precision values for the four test

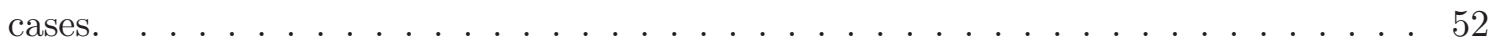

16 Impact of performance tuning on the base similarity recall values for the four test

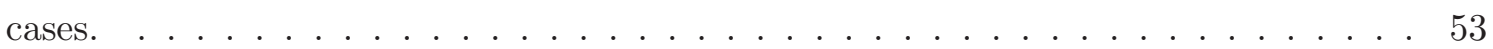

17 Runtime before and after performance tuning for the DSI for the four test cases. . . 54

18 Impact of performance tuning on the DSI precision values for the four test cases. . . 55

19 Impact of performance tuning on the $D S I$ recall values for the four test cases. . . . . 56

20 Runtime before and after performance tuning for the $S S C$ for the four test cases. . . 57

21 Impact of performance tuning on the $S S C$ precision values for the four test cases. . . 58

22 Impact of performance tuning on the $S S C$ recall values for the four test cases. . . . . 59 